\title{
Symmetry and phase-locking in a ring of pulse-coupled oscillators with distributed delays
}

\author{
P.C. Bressloff*, S. Coombes \\ Nonlinear and Complex Systems Group, Department of Mathematical Sciences, Loughborough University, Loughborough, \\ Leicestershire LE11 3TU, UK \\ Received 8 April 1998; accepted 16 September 1998 \\ Communicated by J.P. Keener
}

\begin{abstract}
Phase-locking in a ring of pulse-coupled integrate-and-fire oscillators with distributed delays is analysed using group theory. The period of oscillation of a solution and those related by symmetry is determined self-consistently. Numerical continuation of maximally symmetric solutions in characteristic system length and timescales yields bifurcation diagrams with spontaneous symmetry breaking. The stability of phase-locked solutions is determined via a linearisation of the oscillator firing map. In the weak-coupling regime, averaging leads to an effective phase-coupled model with distributed phase-shifts and the analysis of the system is considerably simplified. In particular, the collective period of a solution is now slaved to the relative phases. For odd numbered rings, spontaneous symmetry breaking can lead to a change of stability of a travelling wave state via a simple Hopf bifurcation. The resulting non-phase-locked solutions are constructed via numerical continuation at these bifurcation points. The corresponding behaviour in the integrate-and-fire system is explored with simulations showing bifurcations to quasiperiodic firing patterns. (c)1999 Elsevier Science B.V. All rights reserved.
\end{abstract}

Keywords: Phase-locking; Pulse-coupled oscillators; Josephson junction; Spiking neurons; Symmetry

\section{Introduction}

The dynamics of coupled nonlinear oscillators has application in many fields of natural science [1,2]. Recent experimental and theoretical interest has focused upon Josephson junctions [3,4], lasers [5], oscillatory chemical reactions [6], heart pacemaker cells [7], central pattern generators [8] and cortical neural oscillators [9]. Typically either small amplitude oscillators near a Hopf bifurcation have been considered $[2,10]$ or a weak coupling of limit cycle oscillators has been utilised. In the latter case, invariant manifold theory [11] and averaging theory [12] can be used to reduce the model to a system of phase equations (see, for example, $[8,13-16]$ ) in which the relative phase between oscillators is the relevant dynamical variable. For certain physical models such as Josephson junction and laser arrays, the dynamics may be expressed in terms of coupled phase variables from the outset. In these cases

\footnotetext{
${ }^{*}$ Corresponding author; E-mail: p.c.bressloff@ boro.ac.uk
} 
the method of averaging can be used for weak coupling provided that, in an appropriate coordinate frame, relative phases evolve on a slow timescale compared to the natural frequency of oscillation in the uncoupled limit. This technique has been applied to globally coupled oscillators [17] and has elucidated the integrable structure of the dissipative, overdamped resistively loaded Josephson array [4,18]. In neural models the effective phase interaction may be regarded as a convolution of the post-synaptic current and some neuronal response function over one period of oscillation. This response function can be obtained from experimental data or constructed directly from the single neuron dynamics. Indeed this has been performed for the Hodgkin-Huxley model [19] and discussed in general for networks of neurons with the so-called type I or II response [20].

In many applications the oscillators are identical, dissipative and the coupling is symmetric. Under such circumstances one can exploit the symmetry of the system to determine generic features of the dynamics such as the emergence of certain classes of solutions due to symmetry breaking bifurcations. Moreover, symmetries have been shown to underly pathological dynamics such as structurally stable heteroclinic connections [21]. Group theoretic methods have been used to study both small amplitude oscillators on a ring near Hopf bifurcation [22], and weakly coupled oscillators under phase-averaging [21,23]. Symmetry arguments have also been used to construct central pattern generators for animal gaits [24] and to establish the existence of periodic orbits in Josephson junction series arrays [25].

Most work to date on the dynamics of coupled oscillator arrays has assumed that the interaction between oscillators depends continuously on their state variables. This smoothness of interaction is absent for oscillators that communicate with sudden, pulse-like discharges. Such interactions are of special interest for neural systems where post-synaptic potentials are induced by the spiking or firing of pre-synaptic neurons. The integrate-and-fire model (see [26] for a review) may be regarded as a reduction of the Hodgkin-Huxley model capable of generating realistic spike trains [27]. This model is of particular interest not only because it is more amenable to analysis than conductance based models but because it has connections with the physics of self-organised criticality [28-30] and $1 / f$ noise [31]. Nevertheless, concrete results concerning the dynamics of integrate-and-fire oscillator networks are still relatively rare and have mainly been restricted to the case of globally coupled arrays. For example, a rigorous analysis of globally coupled integrate-and-fire oscilators using return maps demonstrates the existence of stable phase-locked solutions for instantaneous excitatory coupling [32]. In addition, mean field theory has been applied to large networks in order to study the effects of non-instantaneous coupling common to many neural systems with axonal and synaptic delays [33-36].

In this paper, we present the first comprehensive application of group theoretic and averaging methods to the study of the dynamics of pulse-coupled oscillator networks. For concreteness, we consider a ring of integrate-and-fire oscillators with spatially structured patterns of delayed connections. The integrate-and-fire model evolves according to a linear time-delayed ordinary differential equation until reaching some threshold, whereupon the state variable is instantaneously reset to some pre-defined level. The discontinuous and time-delayed nature of this model allows for extremely rich dynamical behaviour, but at the same time simplifies considerably when one considers the class of frequency-locked solutions in which all the oscillators fire with a common period. This encompasses all phaselocked solutions including, for example, synchronous and travelling wave states. Solutions are determined by a set of algebraic equations involving the relative phases of the oscillators and the collective frequency of oscillation. Importantly, these equations have the same formal structure as those obtained using phase-reduction techniques (see later), but are valid for arbitrary values of the coupling. This suggests the use of various methods previously applied to systems of weakly coupled limit cycle oscillators.

We begin by showing how group theory can be used to classify all possible phase-locked solutions with the collective period determined self-consistently (Section 2). A methodology for constructing solutions from some maximally symmetric set is presented based upon the principle of spontaneous symmetry breaking previously applied to smoothly coupled systems [22]. The linear stability of phase-locked solutions is shown to be readily handled in terms of perturbations of the firing times along analogous lines to van Vreeswijk [36] and Gerstner et al [37]. In Section 3 we present a number of numerical examples for rings of integrate-and-fire oscillators with delayed interactions pertaining to neural systems based upon simple models of axonal communication and synaptic 
processing. Numerical continuation in system parameters is shown to connect phase-locked solutions with differing symmetry groups. However, bifurcations from phase-locked states to non-phase-locked states are, in general, not amenable to such a combination of group theory and numerical continuation. We investigate more general dynamical phenomena by direct numerical integration of the equations of motion. In particular, we establish the occurrence of a discrete Hopf bifurcation in the firing times leading to quasiperiodic variations of the inter-spike intervals on invariant circles. Moreover, in the case of a ring of three IF oscillators, we reveal the existence of a co-dimension one global bifurcation from a travelling wave state to a synchronous state. This type of bifurcation is analogous to the $\mathbf{S}_{3}$ transcritical/homoclinic bifurcation previously studied by Ashwin and co-workers [23] for a system of three weakly coupled Van der Pol oscillators.

In Section 4 a nonlinear transform is used to express the dynamics of the ring of integrate-and-fire oscillators in terms of a set of time-dependent absolute phase variables. The method of averaging is then applied in the weak coupling limit so that the dynamics may be expressed in terms of relative phases on a hypertorus. The effective frequency of oscillation is now slaved to the relative phase of solutions and time delays in the interactions reduce to phase-shifts. The weakly coupled phase model has the same underlying symmetry group as the system of pulse-coupled integrate-and-fire oscillators and once again we exploit this to construct solutions. Moreover, the differentiability of the interaction functions in the phase-coupled model allows one to determine local stability in terms of the eigenvalues of some Jacobian. The Floquet exponents of a periodic orbit show that the condition for linear stability is equivalent to that of the integrate-and-fire system in the weak-coupling regime. We show that for finite size networks and sufficiently small coupling, if there exists a stable or unstable (hyperbolic) phase-locked solution of the phase-coupled model then there exists a corresponding solution of the integrate-and-fire system of the same stability type. In contrast to the integrate-and-fire system, continuation from phase-locked solutions to limit cycles on a hypertorus can be performed at Hopf bifurcation points.

Finally in Section 5 we summarise our findings and discuss extensions to networks with a distribution of frequencies and lacking periodic boundary conditions. (Note that a preliminary report of our work appeared elsewhere [38].)

\section{Integrate-and-fire model}

Consider a circular array of $N$ identical pulse-coupled integrate-and-fire (IF) oscillators labelled $n=1, \ldots, N$ (see Fig. 1 ). Let $U_{n}(t)$ denote the state variable of the $n$th oscillator at time $t$. Suppose that $U_{n}(t)$ satisfies the set of coupled equations:

$$
\frac{\mathrm{d} U_{n}(t)}{\mathrm{d} t}=f\left(U_{n}(t)\right)+\epsilon \sum_{m=1}^{N} \int_{0}^{\infty} J_{m}(\tau) E_{n+m}(t-\tau) \mathrm{d} \tau
$$

supplemented by the reset conditions

$$
U_{n}\left(t^{+}\right)=0 \quad \text { whenever } U_{n}(t)=1 .
$$

Here $E_{m}(t)$ represents the train of pulses transmitted from the $m$ th oscillator at time $t$ and $J_{m}(\tau)$ represents a distribution of delayed connections from the $n$th to the $(n+m)$ th oscillator of the array. (All subscripts $n, m$ are taken as modulo $N$.) The strength of the interactions is determined by the coupling parameter $\epsilon, \epsilon>0$. We shall assume that $J_{n}(\tau)=J_{N-n}(\tau)$ and $J_{n}(\tau)>0$ for all $n, \tau$ so that the network has symmetric excitatory connections. It follows that the underlying symmetry of the ring of coupled oscillators is $\mathbf{D}_{N}$ (cyclic permutations and reflections of the ring). In the special case of global and homogeneous coupling the symmetry is given by the full permutation group $\mathbf{S}_{n}$. for the moment, we shall take $f$ to be a linear function $f\left(U_{n}\right)=-U_{n}+I$ for some constant bias $I, I>1$.

We restrict attention to periodic solutions of Eqs. (1) and (2) in which every oscillator resets or fires with the same period $T$. This period must be determined self-consistently. The state of each oscillator can then be characterised 


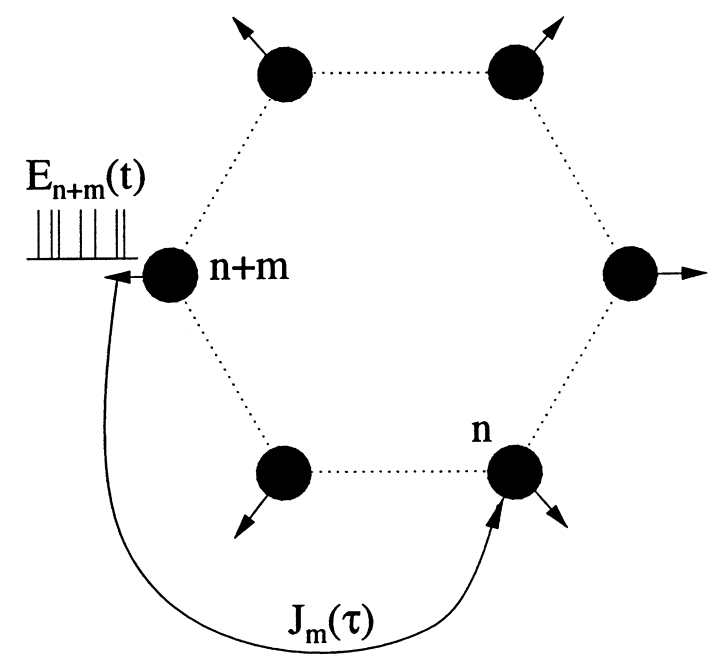

Fig. 1. Basic interaction schematic for a ring of pulse-coupled integrate-and-fire oscillators with distributed delays $J_{m}(\tau)$ and output spike trains $E_{n}(t)$.

by a constant phase $\phi_{n} \in \mathbb{R} \backslash \mathbb{Z}$. We represent the set of $N$ phases by the vector $\Phi=\left(\phi_{1}, \ldots, \phi_{N}\right) \in \mathbb{T}^{N}$, where $\mathbb{T}^{N}$ denotes the $N$-torus. Neglecting the shape of an individual pulse, the resulting spike train is

$$
E_{n}(t)=\sum_{j=-\infty}^{\infty} \delta\left(t-j T+\phi_{n} T\right) \equiv E\left(t+\phi_{n} T\right)
$$

where the firing times of the $n$th oscillator are $\left(j-\phi_{n}\right) T$. Generalizing the analysis of two IF oscillators in [39], we integrate Eq. (1) over the interval $t \in\left(-T \phi_{n}, T-T \phi_{n}\right)$ and incorporate the reset conditon (2) by setting $U_{n}\left(-\phi_{n} T\right)=0$ and $U_{n}\left(T-\phi_{n} T\right)=1$. This leads to the $N$ equations:

$$
1=\left(1-\mathrm{e}^{-T}\right) I+\epsilon \sum_{m=1}^{N} K_{m}\left(\phi_{n+m}-\phi_{n}, T\right), \quad n=1, \ldots, N,
$$

where

$$
K_{m}(\phi, T)=\mathrm{e}^{-T} \int_{0}^{T} \mathrm{e}^{t} \hat{J}_{m}(t+\phi T) \mathrm{d} t
$$

may be regarded as an effective interaction function, with coupling strength $\epsilon$, and

$$
\hat{J}_{m}(t)=\sum_{j=0}^{\infty} J_{m}(t+j T)
$$

for $0 \leq t<T$ and $\hat{J}_{m}(t)$ is extended outside this range by making it a periodic function of $t$. After choosing some reference oscillator, Eqs. $(4)$ determine $(N-1)$ relative phases and the period $T$.

\subsection{A group theoretic approach}

In many applications one comes across oscillators that are identical, dissipative (non-Hamiltonian) and have some symmetry in their coupling. Three types of symmetry often occur; the cyclic group $\mathbf{Z}_{N}$ (the symmetry of a directed $N$-gon), the dihedral group $\mathbf{D}_{N}$ (the symmetry of a regular $N$-gon) and the symmetric group $\mathbf{S}_{N}$ (all permutations of 
$N$ objects). The seminal work of Turing [40] discusses rings of oscillators with $\mathbf{D}_{N}$ symmetry and weakly coupled rings of such oscillators near a Hopf bifurcation have been studied in detail by Ermentrout [41] using perturbation and numerical methods. More recently, the technological importance of large arrays of fully connected coupled Josephson junction oscillators has focused attention upon oscillator networks with $\mathbf{S}_{N}$ symmetry [3].

The system of equation (4) is invariant under the action of the group $\Gamma=\mathbf{D}_{N} \times \mathbf{S}^{1}$. That is, if $\Phi=\left(\phi_{1}, \ldots, \phi_{N}\right)$ is a solution of Eqs. (4), then so is $\sigma \Phi$ for all $\sigma \in \Gamma$. The dynamics of weakly phase-coupled oscillators with this specific group symmetry has previously been discussed in [21]. In contrast we consider a time-independent system of algebraic equations that determine the phase and period of a ring of IF oscillators with arbitrary coupling strength. We take the generators of $\mathbf{D}_{N}$ to be $\{\gamma, \kappa\}$ with $[\gamma \Phi]_{n}=\phi_{n+1}$ and $[\kappa \Phi]_{n}=\phi_{N-n+2}$. The additional $\mathbf{S}^{1}$ symmetry corresponds to constant phase shifts $\phi_{n} \rightarrow \phi_{n}+\delta$ and is a consequence of the fact that Eqs. (4) depend on phase differences. Hence the original system of IF oscillators possess a symmetry combining geometric transformations of oscillators in the ring with time-translations in the form of oscillator phase shifts. The symmetry of the group $\Gamma$ is expressed by the action

$$
[(\mu, \nu) \Phi]_{n}=\nu \Phi_{\mu(n)}
$$

for $(\mu, v) \in \mathbf{D}_{N} \times \mathbf{S}^{1}$, where $\nu \Phi=\left(\phi_{1}+v, \ldots, \phi_{N}+v\right)$. Any solution of Eqs. (4) determines $\phi$ (up to an arbitrary phase-shift) and the period $T=T(\Phi)$ such that $T(\sigma \Phi)=T(\Phi)$ for all $\sigma \in \Gamma$.

The existence of an underlying symmetry group allows one to systematically explore the different classes of solutions to Eqs. (4) and their associated bifurcations. In order to investigate this issue further, it is useful to introduce a few simple definitions from group theory. (For a general account of symmetries in bifurcation theory, see Golubitsky et al. [22].) The symmetries of any particular solution $\Phi$ form a subgroup called the isotropy subgroup of $\Phi$ defined by

$$
\Sigma_{\Phi}=\{\sigma \in \Gamma: \sigma \Phi=\Phi\}
$$

More generally, we say that $\Sigma$ is an isotropy subgroup of $\Gamma$ if $\Sigma=\Sigma_{\Phi}$ for some $\Phi \in \mathbb{T}^{N}$. We adopt the practice that isotropy subgroups are defined up to some conjugacy. A group $\Sigma$ is conjugate to a group $\hat{\Sigma}$ if there exists $\sigma \in \Gamma$ such that $\hat{\Sigma}=\sigma^{-1} \Sigma \sigma$. The fixed-point subspace of an isotropy subgroup $\Sigma$, denoted by $\operatorname{Fix}(\Sigma)$, is the set of points $\Phi \in \mathbb{T}^{N}$ that are invariant under the action of $\Sigma$ :

$$
\operatorname{Fix}(\Sigma)=\left\{\Phi \in \mathbb{T}^{N}: \sigma \Phi=\Phi \forall \sigma \in \Sigma\right\} \text {. }
$$

Finally, the group orbit through a point $\Phi$ is

$$
\Gamma \Phi=\{\sigma \Phi: \sigma \in \Gamma\} .
$$

If $\Phi$ is a solution to Eqs. (4) then so are all other points of the group orbit. One can now adopt a strategy that restricts the search for solutions of Eqs. (4) to those that are fixed points of a particular isotropy subgroup. In general, if a dynamical system is invariant under some symmetry group $\Xi$ and has a solution that is a fixed point of the full symmetry group then we expect a loss of stability to occur upon variation of one or more system parameters. Typically such a loss of stability will be associated with the occurrence of new solution branches with isotropy subgroups $\Sigma$ smaller than $\Xi$. One says that the solution has spontaneously broken symmetry from $\Xi$ to $\Sigma$. Instead of a unique solution with the full set of symmetries $\Xi$, a set of symmetrically related solution (orbits under $\Xi$ modulo $\Sigma$ ) each with symmetry group (conjugate to) $\Sigma$ is observed. In many physical systems the subgroups $\Sigma$ are maximal isotropy subgroups. $\Sigma$ is maximal if $\operatorname{dim} \operatorname{Fix}(\Sigma)=1$. The equivariant branching lemma (see [22]) guarantees that, with certain condition on $\Sigma$, if $\operatorname{dim} \operatorname{Fix}(\Sigma)=1$, then a unique branch of solutions with isotropy subgroup $\Sigma$ does indeed exist. In the case of our particular system, there are no solutions that are fixed points of the full symmetry group $\mathbf{D}_{N} \times \mathbf{S}^{1}$. Therefore, we shall be interested in spontaneous symmetry breaking from maximally symmetric solutions to solutions with smaller isotropy subgroups.

The isotropy subgroups of $\Gamma=\mathbf{D}_{N} \times \mathbf{S}^{1}$ and their fixed-point spaces for the system of equations (4), are shown in Table 1. The fixed-point spaces consist of $m$ blocks of $k$ adjacent oscillators, having the same period and 
Table 1

The isotropy subgroups, $\Sigma$, of $\Gamma=\mathbf{D}_{N} \times \mathbf{S}^{1}$.

\begin{tabular}{|c|c|c|}
\hline$\Sigma$ & $\operatorname{Fix}(\Sigma)$ & $\operatorname{dim} \operatorname{Fix}(\Sigma)$ \\
\hline \multicolumn{3}{|l|}{$k=1$} \\
\hline $\mathbf{D}_{N}$ & $(\phi, \ldots, \phi)$ & 1 \\
\hline $\mathbf{D}_{N}(0,1 / 2)$ & $(\phi, \bar{\phi}, \phi, \ldots, \bar{\phi}), N$ even & 1 \\
\hline $\mathbf{Z}_{N}(\beta)$ & $(\phi, \phi+\beta, \phi+2 \beta, \ldots, \phi+(N-1) \beta), n_{b} \in\{1, \ldots,(N-1)\}$ & 1 \\
\hline \multicolumn{3}{|c|}{ 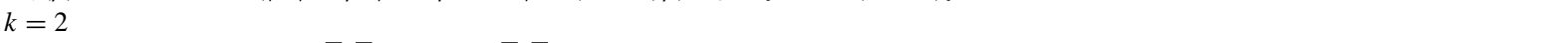 } \\
\hline $\mathbf{D}_{N / 2}$ & $(\phi, \phi, \bar{\phi}, \bar{\phi}, \ldots, \phi, \phi, \bar{\phi}, \bar{\phi}), N=0 \bmod 4$ & 1 \\
\hline $\mathbf{D}_{N / 2}(\kappa)$ & $\left(\phi_{1}, \phi_{2}, \phi_{1}, \phi_{2}, \ldots, \phi_{1}, \phi_{2}\right)$ & 2 \\
\hline $\mathbf{Z}_{N / 2}(\beta)$ & $\left(\phi_{1}, \phi_{2}, \phi_{1}+2 \beta, \phi_{2}+2 \beta, \ldots, \phi_{1}+(N-2) \beta, \phi_{2}+(N-2) \beta\right), n_{b} \in\{1, \ldots,[N / 2]\}$ & 2 \\
\hline \multicolumn{3}{|c|}{ 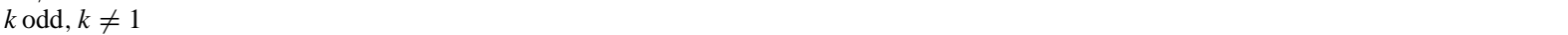 } \\
\hline $\mathbf{D}_{m}$ & $\left(\phi_{1}, \phi_{2}, \phi_{3}, \phi_{2}, \phi_{1}, \ldots, \phi_{1}, \phi_{2}, \phi_{3}, \phi_{2}, \phi_{1}\right)$ & $(k+1) / 2$ \\
\hline $\mathbf{D}_{m}(0,1 / 2)$ & $\left(\phi_{1}, \phi_{2}, \phi_{3}, \phi_{2}, \phi_{1}, \ldots, \overline{\phi_{1}}, \overline{\phi_{2}}, \overline{\phi_{3}}, \overline{\phi_{2}}, \overline{\phi_{1}}\right)$, m even & $(k+1) / 2$ \\
\hline $\mathbf{Z}_{m}$ & $\left(\phi_{1}, \phi_{2}, \phi_{3}, \phi_{4}, \phi_{5}, \ldots, \phi_{1}, \phi_{2}, \phi_{3}, \phi_{4}, \phi_{5},\right)$ & $k$ \\
\hline $\mathbf{Z}_{m}(\beta)$ & $\left(\phi_{1}, \ldots, \phi_{5}, \phi_{1}+5 \beta, \ldots, \phi_{5}+5 \beta, \ldots, \phi_{1}+(N-5) \beta, \ldots, \phi_{5}+(N-5) \beta\right), n_{b} \in\{1, \ldots, m\}$ & $k$ \\
\hline \multicolumn{3}{|c|}{ 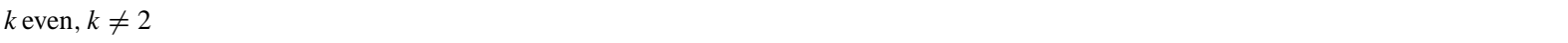 } \\
\hline $\mathbf{D}_{m}(\kappa)$ & $\left(\phi_{1}, \phi_{2}, \phi_{3}, \phi_{4}, \phi_{3}, \phi_{2}, \ldots, \phi_{1}, \phi_{2}, \phi_{3}, \phi_{4}, \phi_{3}, \phi_{2}\right)$ & $k / 2+1$ \\
\hline $\mathbf{D}_{m}(\kappa \gamma)$ & $\left(\phi_{1}, \phi_{2}, \phi_{3}, \phi_{3}, \phi_{2}, \phi_{1} \ldots, \phi_{1}, \phi_{2}, \phi_{3}, \phi_{3}, \phi_{2}, \phi_{1}\right)$ & $k / 2$ \\
\hline $\mathbf{D}_{m}(1 / 2,1 / 2)$ & $\left(\phi_{1}, \phi_{2}, \phi_{3}, \overline{\phi_{3}}, \overline{\phi_{2}}, \overline{\phi_{1}} \ldots, \phi_{1}, \phi_{2}, \phi_{3}, \overline{\phi_{3}}, \overline{\phi_{2}}, \overline{\phi_{1}}\right)$ & $k / 2$ \\
\hline $\mathbf{D}_{m}(0,1 / 2)$ & $\left(\phi_{1}, \phi_{2}, \phi_{3}, \phi_{3}, \phi_{2}, \phi_{1} \ldots, \overline{\phi_{1}}, \overline{\phi_{2}}, \overline{\phi_{3}}, \overline{\phi_{3}}, \overline{\phi_{2}}, \overline{\phi_{1}}\right), m$ even & $k / 2$ \\
\hline $\mathbf{Z}_{m}$ & $\left(\phi_{1}, \phi_{2}, \phi_{3}, \phi_{4}, \ldots, \phi_{1}, \phi_{2}, \phi_{3}, \phi_{4}\right)$ & $k$ \\
\hline $\mathbf{Z}_{m}(\beta)$ & $\left(\phi_{1}, \ldots, \phi_{4}, \phi_{1}+4 \beta, \ldots, \phi_{4}+4 \beta, \ldots, \phi_{1}+(N-4) \beta, \ldots, \phi_{4}+(N-4) \beta\right), n_{b} \in\{1, \ldots, m\}$ & $k$ \\
\hline
\end{tabular}

There are $m$ blocks of $k$ adjacent oscillators in the fixed-point spaces, where $N=m k, \bar{\phi}=\phi+1 / 2$, and $\beta=n_{b} / N$.

amplitude, where $m k=N$ runs through all binary factorisations of $N$. The elements of $\mathbf{D}_{N}$ may be regarded as spatial symmetries and elements of $\mathbf{S}^{1}$ as acting on solutions by phase shift. All proper isotropy subgroups of $\Gamma$ are twisted subgroups so that $(\mu, v) \in \Gamma$ may be written as $(\mu, \nu(\mu))$. Spatial symmetries arise for $v(\mu)=0$ and spatial symmetries combined with phase-shifts for $v(\mu) \neq 0$. A method for constructing the (twisted) isotropy subgroups of $\mathbf{D}_{N} \times \mathbf{S}^{1}$ exists based upon knowledge of subgroups of $\mathbf{D}_{N}$. Without reproducing details (see [21]), we list the isotropy subgroups of $\Gamma$ as follows. $\mathbf{D}_{m}(\kappa)$ and $\mathbf{D}_{m}(\kappa \gamma)$ denote the subgroups of $\mathbf{D}_{N}$ with generators $\left\{\gamma^{k}, \kappa\right\}$ and $\left\{\gamma^{k}, \kappa \gamma\right\}$, respectively. The generators of the cyclic group $\mathbf{Z}_{m} \subset \mathbf{D}_{m}$ are simple $\left\{\gamma^{k}\right\}$. The groups $\mathbf{D}_{m}(0,1 / 2), \mathbf{D}_{m}(1 / 2,1 / 2)$ and $\mathbf{Z}_{m}(\beta)$ are all twisted subgroups of $\Gamma$ with generators $\left\{\left(\gamma^{k-1} \kappa, 0\right),(\kappa \gamma, 1 / 2)\right\}(m$ even $),\left\{\left(\gamma^{k-1} \kappa, 1 / 2\right),(\kappa \gamma, 1 / 2)\right\}$ ( $k$ even) and $\left\{\gamma^{k}, k \beta\right\}\left(\beta=n_{b} / N, n_{b} \in\{1, \ldots, m\}\right)$. The phases $\phi_{1}, \ldots, \phi_{k}$ determine the state of the system, and the dimension of the fixed point space is the number of independent phases within this block. $\operatorname{If} \operatorname{dimFix}(\Sigma)=d$, then the $N$ equations of [4] reduce to $d$ independent equations, which leads to a considerable simplification when $d \ll N$. In particular, if $d=1$, then a solution is guaranteed to exist by the underlying symmetry. This is nothing more than a restatement of the equivariant branching lemma to the effect that solutions exist for isotropy with one-dimensional fixed-point subspaces.

Examples of these maximally symmetric solutions with $d=1$ are the synchronous or in-phase solution, $\phi_{n}=\phi$ for all $n$, and travelling wave solutions, $\phi_{n}=\phi+n \beta$ with $\beta=n_{b} / N, n_{b}=1, \ldots, N-1$. For even $N$ one also has alternating anti-phase solutions of the form $(\phi, \bar{\phi}, \phi, \bar{\phi}, \ldots)$. Here $\phi$ is an arbitrary phase and $\bar{\phi}=\phi+1 / 2$. In these cases $(d=1)$, Eqs. (4) reduces to one equation that determines the period $T$. For example, substituting $\phi_{n}=n \beta$ into Eqs. (4) gives the following implicit equation for $T$ :

$$
1=\left(1-\mathrm{e}^{-T}\right) I+\epsilon \sum_{m} K_{m}(m \beta, T)
$$

The corresponding travelling wave solution satisfies $U_{n}(t)=U(t / T+n \beta)$ where $U(t)=U(t+T)$ is some periodic waveform. As mentioned above we have no general method for answering the question as to whether there exists a branch of solution to algebraic systems of the type (4) for a given isotropy subgroup, except for the maximally symmetric case with $d=1$. However, we shall show through numerical examples in Section 3 that maximally 
symmetric solutions often bifurcate into solutions that have a smaller isotropy group when some system parameter is varied. Such a parameter may be taken to be a characteristic length or timescale of the coupled oscillators, for example, the range of interactions, a discrete communication delay time for pulses, or, for neural systems, a typical distance of synapses from the soma in dendritic processing. All of these features may be modelled with appropriate choices of the distribution $J_{m}(\tau)$ (see Section 2.3).

\subsection{Stability of phase-locked solutions}

In general it is possible to construct an implicit map of the firing times for the system of integrate-and-fire oscillators with dynamics given by Eq. (1) from the reset conditions (2). Consider perturbations of the regular firing pattern $T_{j}^{n} \equiv\left(j-\phi_{n}\right) T$ such that $T_{j}^{n} \rightarrow T_{j}^{n}+\delta_{j}^{n}[36,37]$. The linear stability of the phase-locked solution, denoted by $\Phi$, can be determined from a linearised map taking the explicit form

$$
A_{n}(\Phi, T)\left[\delta_{k+1}^{n}-\delta_{k}^{n}\right]+B_{n}(\Phi, T) \delta_{k}^{n}=\sum_{m=1}^{N} \sum_{j=F_{k+1}(m, n)}^{\infty} a_{n m, j}(\Phi, T) \delta_{k-j}^{m+n},
$$

where

$$
\begin{aligned}
& F_{k}(m, n)=-1 \quad \text { if } T_{k}^{n}+\delta_{k}^{n}>T_{k}^{n+m}+\delta_{k}^{n+m}, \\
& F_{k}(m, n)=0 \quad \text { if } T_{k}^{n}+\delta_{k}^{n}<T_{k}^{n+m}+\delta_{k}^{n+m} .
\end{aligned}
$$

The function $F_{k}(m, n)$ is necessary to ensure that the map (12) is a retarded difference equation. The coefficients $A_{n}(\Phi, T), B_{n}(\Phi, T)$ and $a_{n m, j}(\Phi, T)$ may be determined by expanding $U_{n}\left(T_{k+1}^{n}+\delta_{k+1}^{n}\right)=1$ with $U_{n}\left(T_{k}^{n}+\delta_{k}^{n}\right)=0$ to first order in $\delta_{j}^{n}$. In this instance,

$$
\begin{aligned}
& A_{n}(\Phi, T)=I-1+\epsilon \sum_{m} \hat{J}_{m}\left(\left(\phi_{n+m}-\phi_{n}\right) T\right), \\
& B_{n}(\Phi, T)=\frac{\epsilon}{T} \sum_{m} K_{m}^{\prime}\left(\phi_{n+m}-\phi_{n}, T\right), \\
& a_{n m, j}(\Phi, T)=\frac{\epsilon}{T} \int_{0}^{T} \mathrm{e}^{t-T} J_{m}^{\prime}\left(t+\left(j+\phi_{n+m}-\phi_{n}\right) T\right) \Theta\left(t+\left(j+\phi_{n+m}-\phi_{n}\right) T\right) \mathrm{d} t,
\end{aligned}
$$

where' indicates differentiation with respect to $\phi$, and $\Theta(x)=1$ if $x \geq 0$ and is zero otherwise. Substitution into the linearised map (12) a solution of the form $\delta_{k}^{n}=\lambda^{k} \delta_{n}$, for $\delta_{n} \in \mathbb{R}$ and $\lambda \in \mathbb{C}$, yields the eigenvalue equation:

$$
(\lambda-1) A_{n}(\Phi, T) \delta_{n}+B_{n}(\Phi, T) \delta_{n}=\sum_{m=1}^{N} \tilde{a}_{n m}(\lambda, \Phi, T) G_{n m}(\lambda) \delta_{n+m},
$$

where

$$
\tilde{a}_{n m}(\lambda, \Phi, T)=\sum_{j=0}^{\infty} a_{n m, j}(\Phi, T) \lambda^{-j},
$$

and $G_{n m}(\lambda)=\lambda$ if $\phi_{n}<\phi_{n+m}$, and $G_{n m}=1$ if $\phi_{n}>\phi_{n+m}$ on [0,1]. Note that one solution to (17) is given by $\lambda_{0}=1$ with $\delta_{m}=\delta$ all $\mathrm{m}$. This reflects invariance of the dynamics with respect to uniform phase-shifts. The condition for asymptotic stability of a solution is $|\lambda|<1$ for all eigenvalues $\left(\lambda \neq \lambda_{0}\right)$ satisfying Eq. (17).

In general, carrying out a linear stability analysis of phase-locked solutions is a non-trivial task due to the fact that the dynamical system is infinite-dimensional. However, in the weak-coupling limit, $\epsilon \rightarrow 0$, linear stability analysis 
becomes much more tractable since solutions to (17) in the complex $\lambda$-plane will either be in the neighbourhood of the real solution $\lambda=1$ or in the neighbourhood of one of the poles of $\tilde{a}_{n m}(\lambda, \Phi, T)$. These poles all lie inside the unit circle and hence are not important in terms of determining whether or not a phase-locked solution is stable. Therefore, to first-order in $\epsilon$ we set $\lambda=1$ and $T \rightarrow T_{0}=\ln (I /(I-1))$ on the right-hand side of (17) to yield

$$
(\lambda-1) \delta_{n}=\frac{\epsilon}{(I-1) T_{0}} \sum_{m} K_{m}^{\prime}\left(\phi_{n+m}-\phi_{n}, T_{0}\right)\left[\delta_{n+m}-\delta_{n}\right]+\mathrm{O}\left(\epsilon^{2}\right) .
$$

Thus to $\mathrm{O}(\epsilon)$ the spectrum close to $\lambda=1$ coalesces into $N$ distinct points given by the eigenvalues $\lambda_{p}=1+\Gamma_{p}, p=$ $0, \ldots,(N-1)$ with $\Gamma_{0}=0$. Here $\Gamma_{p}, p=0, \ldots,(N-1)$ form the set of eigenvalues of the matrix with components $\hat{\mathcal{K}}_{n m}(\Phi)=\mathcal{K}_{n m}(\Phi)-\delta_{n m} \sum_{k} \mathcal{K}_{n k}(\Phi)$, where

$$
\mathcal{K}_{n m}(\Phi)=\frac{\epsilon}{(I-1) T_{0}} K_{m-n}^{\prime}\left(\phi_{m}-\phi_{n}, T_{0}\right)
$$

The fact that $\Gamma_{0}=0$, with a corresponding eigenvector in the direction of the flow $(1,1, \ldots, 1)$, again shows the symmetry $\left(\mathbf{S}^{1}\right)$ of the system to constant translations of the phases. The condition for stability reduces to the set of $(N-1)$ conditions $\operatorname{Re}\left(\Gamma_{p}\right)<0, p \neq 0$. Take, for example, travelling wave states of the type $\phi_{m}=m \beta, \beta=$ $n_{b} / N$ and $n_{b}=1, \ldots,(N-1)$. The eigenvectors of $\hat{\mathcal{K}}(\Phi)$ are $\left(1, \mathrm{e}^{2 \pi \mathrm{i} p / N}, \mathrm{e}^{4 \pi \mathrm{i} p / N}, \ldots, \mathrm{e}^{2(N-1) \pi \mathrm{i} p / N}\right)$ with the corresponding eigenvalues:

$$
\Gamma_{p}=\frac{\epsilon}{(I-1) T_{0}} \sum_{m} K_{m}^{\prime}\left(m \beta, T_{0}\right)\left[\mathrm{e}^{2 \pi \mathrm{i} m p / N}-1\right]
$$

The above weak coupling stability condition will be rederived in Section 4 in terms of a corresponding phase model obtained by the method of averaging.

Assume that a given phase-locked solution $\Phi$ is stable in the small coupling regime but becomes unstable when $\epsilon$ is increased. If a single real eigenvalue $\lambda \neq \lambda_{0}$ crosses $\lambda=1$ at a critical value of the coupling $\epsilon_{\mathrm{c}}$, then the solution $\Phi$ will destabilise via a static bifurcation of the firing times. The bifurcating solutions will correspond to new phase-locked states and the oscillators will remain 1:1 frequency-locked. On the other hand, if a complex conjugate pair of eigenvalues $\left(\lambda, \lambda^{*}\right)$ crosses the unit circle, then $\Phi$ will destabilise via a discrete Hopf bifurcation in the firing times leading to the breakdown of 1:1 frequency-locking. This form of destabilisation turns out to play a major role in the formation of complex firing patterns in IF networks, as will be explored in more detail elsewhere $[42,43]$. Here we shall only briefly touch on this important aspect of spike train dynamics, so that we can interpret the numerical results presented in Section 3.

Suppose that at a critical value of the coupling $\epsilon_{\mathrm{c}}$ there exists a complex conjugate pair eigenvalues $\lambda=\mathrm{e}^{ \pm \mathrm{i} \omega_{\mathrm{c}}}$ signalling the onset of a (supercritical) Hopf bifurcation. Set $\omega_{\mathrm{c}} \equiv 2 \pi \beta$ and assume that either $\beta$ is irrational (nonresonant) or $\beta=p / q$ with $p, q$ co-prime integers and $p>4$ (weakly resonant). Then close to the bifurcation point the perturbations $\delta_{k}^{n}$ have the approximate form $\delta_{k}^{n}=r_{n} \cos \left(k \omega+\theta_{n}\right)$ for some constant phase $\theta_{n}$, amplitude $r_{n}=\mathrm{O}(\sqrt{\epsilon})$ and frequency $\omega \approx \omega_{\mathrm{c}}+\mathrm{O}(\epsilon)$. The inter-spike interval $D_{n}(k)=T_{k+1}^{n}-T_{k}^{n}$ between two consecutive firings of the $n$th cell will then satisfy $D_{n}(k) \equiv T+\delta_{k+1}^{n}-\delta_{k}^{n}=T-\tilde{r}_{n} \sin \left(k \omega+\tilde{\theta}_{n}\right)$, where $\tilde{r}_{n}=2 r_{n} \sin (\omega / 2), \tilde{\theta}_{n}=\theta_{n}+\omega / 2$. Hence, the pair $\left(D_{n}(k-1), D_{n}(k)\right)$ lies on the invariant circle

$$
\mathcal{M}_{n}: \theta \mapsto\left(T-\tilde{r}_{n} \sin (\theta-\omega), T-\tilde{r}_{n} \sin (\theta)\right)
$$

with $0 \leq \theta<2 \pi$. (More precicesly, $\mathcal{M}_{n}$ is a projection of an invariant circle existing in the full phase-space of the dynamical system). If $\beta$ is rational, then the resulting sequence of inter-spike intervals on $\mathcal{M}_{n}$ will be periodic in $k$ ( $p: q$ mode-locking). Associated with the weak resonances are Arnold tongues that spread out in parameter space from the points at which $\beta=p / q$. On the other hand, for irrational $\beta$ the sequence of inter-spike intervals will be quasiperiodic on $\mathcal{M}_{n}$. 


\subsection{Forms of delay for neural systems}

A systematic attack on understanding the dynamics of the brain may arise through a study of coupled neural oscillators. Indeed, the nonlinear dynamics of coupled oscillators consisting of biologically plausible neuron models has recently attracted much interest in neurobiology due to the discovery of synchronised oscillations in the cat visual cortex [9]. Moreover, many biological rhythms, ranging from breathing to walking, are programmed in part by central pattern generating (CPG) networks built from coupled neuronal oscillators. The generation and control of rhythmic activity results from a combination of synaptic interactions, intrinsic membrane properties and network connectivity. Guided by the study of small networks, say in the spinal cord of the Lamprey or Xenopus tadpole and other experimentally accessible systems the fundamental properties of neurons that contribute to rhythm generation are being uncovered [44]. Three such properties are axonal communication delays, synaptic processing and the distribution of axo-dendritic synapses on the dendritic tree. Interestingly, the distributed and discrete delays arising from these processes may also play a role in the formation of oscillatory waves observed in such structures as the olfactory cortex $[45,46]$.

If we think of the IF oscillator as a model neuron then forms of discrete and distributed neural delays can be modelled as follows.

\subsubsection{Space-dependent transmission delays}

Space-dependent delays are a natural feature of networks of point processors communicating with finite signal propagation velocities. For example a transmission delay $\tau_{m}$ may increase with separation according to $\tau_{m}=d_{m} / v$, where $v$ is the signal propagation velocity and $d_{m}$ is the distance between any two oscillators in the ring (measured in units of the lattice spacing). That is, $d_{m}=m$ if $m \leq[N / 2]$ and $d_{m}=N-m$ otherwise. In a neural context $\tau_{m}$ may represent the transmission time for propagation of an action potential along a single axon from the $n$th neuron to the $(n+m)$ th neuron. We represent space-dependent communication delays in the form

$$
J_{m}(\tau)=W_{m} P\left(\tau-\tau_{m}\right) \Theta\left(\tau-\tau_{m}\right)
$$

for some $P(\tau)$ and $\tau_{m}$. Throughout we shall take weight distributions $W_{m}$ with $W_{m}=W_{N-m}$ and similarly for $\tau_{m}$.

\subsubsection{Synaptic processing}

The arrival of an action potential at a synapse triggers the release of chemical neurotransmitters that diffuse across the synaptic cleft and bind to protein receptors in the cell membrane of the post-synaptic neuron. This leads to the generation of a post synaptic potential associated with the opening and closing of various ionic channels. A reasonable approximation to the shape of such a potential is the so-called $\alpha$-function [47]:

$$
g(\tau)=\tau \alpha^{2} \exp (-\alpha \tau)
$$

where $\alpha$ is the inverse rise-time. Synaptic processing can be modelled by taking $J_{m}(\tau)=W_{m} g(\tau)$.

\subsubsection{Dendritic processing}

A post-synaptic potential is typically generated at a synapse located on the dendritic tree of a neuron and is thus at some distance from the soma or cell body where action potential generation occurs. The passive membrane properties of the dendrites result in diffusion of the post-synaptic potential along the tree. For simplicity, suppose that the dendrites are represented by an infinite uniform cable with dendritic coordinates $\xi \in \mathbb{R}$ and the soma is at $\xi=0$. Let $V_{n}(\xi, t)$ denote the dendritic potential at position $\xi$ along the cable of the $n$th neuron. Suppose that there is a distribution of axo-dendritic connections from the $n$th to the $(n+m)$ th neuron as specified by the function $W_{m}(\xi)$.

Using standard cable theory [48], Eq. (1) is replaced by the set of equations:

$$
\frac{\mathrm{d} U_{n}(t)}{\mathrm{d} t}=f\left(U_{n}(t)\right)+I_{n}(t)
$$




$$
\frac{\partial V_{n}(\xi, t)}{\partial t}=D \frac{\partial^{2} V_{n}(\xi, t)}{\partial \xi^{2}}-\frac{V_{n}(\xi, t)}{\tau_{s}}+\sum_{m=1}^{N} W_{m}(\xi) E_{n+m}(t)-I_{n}(t),
$$

where $D$ is the diffusion constant and $\tau_{s}$ is the membrane leakage time constant of the cable. The term $I_{n}(t)=$ $\epsilon\left[V_{n}(0, t)-U_{n}(t)\right]$ is the current density flowing to the soma from the cable at $\xi=0$. In order to simplify our analysis we assume that the current $-I_{n}(t)$ in Eq. (26) is negligible compared to the synaptic current. The dendritic potentials appear linearly in Eq. (26) so that they can be handled using a standard Green's function method. The result is the integral equation:

$$
V_{n}(0, t)=\int_{-\infty}^{t} \int_{-\infty}^{\infty} G\left(\xi, t-t^{\prime}\right) \sum_{m=1}^{N} W_{m}(\xi) E_{n+m}\left(t^{\prime}\right) \mathrm{d} \xi \mathrm{d} t^{\prime},
$$

where

$$
G(\xi, t)=\frac{1}{\sqrt{4 \pi D t}} \exp \left(-\frac{\xi^{2}}{4 D t}\right) \exp \left(-\frac{t}{\tau_{s}}\right)
$$

is the fundamental solution of the cable equation on the real line. Substituting Eq. (27) into (26), and redefining the function $f\left(U_{n}\right)$ to include the term $-\epsilon U_{n}$, yields Eq. (1) with an effective distribution of delays of the form

$$
J_{m}(\tau)=\int_{-\infty}^{\infty} W_{m}(\xi) G(\xi, \tau) \mathrm{d} \xi
$$

\section{Numerical examples}

In this section we provide some illustrative examples of spontaneous symmetry breaking. We concentrate on bifurcations from maximally symmetric isotropy subgroups $\Sigma$ with $\operatorname{dim} \operatorname{Fix}(\Sigma)=1$ for the reasons given in Section 2.1. Numerical continuation of solutions is performed with the aid of XPP [49] in parameters that describe the distributions discussed in Section 2.3. Moreover, we present a direct integration of the equations of motion (1) to illustrate the variation of the inter-spike interval in certain parameter regimes. For simplicity we only consider axonal and synaptic delays.

Example 1. $(N=2)$. Two coupled oscillators suffice to uncover the influence of distributed delays upon synchronisation [39,50-52] and to exhibit the phenomenon of spontaneous symmetry breaking. The underlying symmetry is $\mathbf{Z}_{2} \times \mathbf{S}^{1}$ for a connection between the pair of oscillators of the form $J(\tau)=g\left(\tau-\tau_{\mathrm{d}}\right) \Theta\left(\tau-\tau_{\mathrm{d}}\right)$, where $g(\tau)$ is the $\alpha$-function of Eq. (24) and $\tau_{\mathrm{d}}$ is a simple transmission delay. For $N=2$, Eqs. (4) become

$$
1=\left(1-\mathrm{e}^{-T}\right) I+\epsilon K\left( \pm \phi-\tau_{\mathrm{d}} / T, T\right)
$$

where $\phi=\phi_{2}-\phi_{1}$,

$$
K(\phi, T)=\mathrm{e}^{-T} \int_{0}^{T} \mathrm{e}^{t} \hat{J}(t+\phi T) \mathrm{d} T
$$

and

$$
\hat{J}(t)=\sum_{j=0}^{\infty} g(t+j T)=\frac{\alpha^{2} \mathrm{e}^{\alpha t}}{1-\mathrm{e}^{-\alpha T}}\left[t+\frac{T \mathrm{e}^{-\alpha T}}{\left(1-\mathrm{e}^{-\alpha T}\right)}\right]
$$

for $0 \leq t<T$. The pair of equations (30) reduce to one independent equation (for the period $T$ ) in the case of the synchronous solution $\phi=0$ (or equivalently $\phi=1$ ) and the anti-phase solution $\phi=1 / 2$. Both of these are 


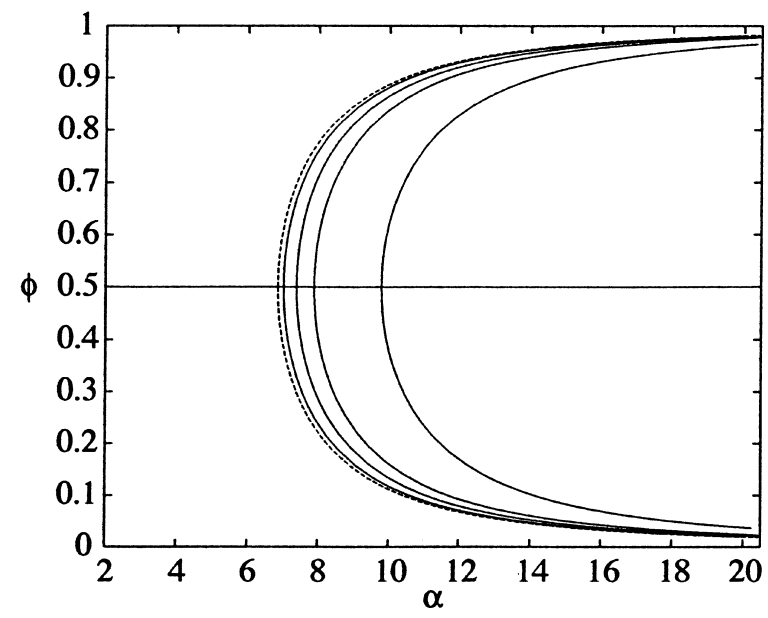

Fig. 2. Relative phase $\phi=\phi_{2}-\phi_{1}$ in the IF model for $N=2$ as a function of the distributed delay parameter $\alpha$ is shown with solid lines for $\epsilon=0.01,0.05,0.1,0.25$ with $t_{\mathrm{d}}=0$ and $I=2$. In each case, a stable anti-phase state undergoes a bifurcation at a critical value of $\alpha$ (which increases with $\epsilon$ ), where it becomes unstable and two additional stable solutions $\phi, 1-\phi$ are created. The dashed curve shows the bifurcation branches in the limiting case of the weakly coupled phase-interaction picture.

guaranteed to exist by the symmetry of the problem. In Fig. 2, we show how an additional pair of solutions $\{\phi, 1-\phi\}$ with $0<\phi<1 / 2$ bifurcates from the anti-phase solution as the parameter $\alpha$ is varied. (The fact that $1-\phi$ is a solution when $\phi$ is a solution is again a consequence of the underlying symmetry, that is, they lie on the same group orbit). A special feature of two oscillators is that one can determine a simple necessary condition for stability of the above periodic solutions [39]. First, following the same procedure as in the derivation of Eqs. (30), it is simple to establish that $U_{2}(T-\phi T)=1-K_{-}(\phi, T)$ where $K_{-}(\phi, T)=K(\phi, T)-K(-\phi, T)$. Suppose that $\phi$ is slightly larger than a fixed point solution $\bar{\phi}$ of Eqs. (30). Then, oscillator 2 should fire later to restore the correct value of $\phi$ if such a solution is to be locally stable. This requires that $U_{2}(T-\phi T)$ should be smaller than the threshold 1 or equivalently that $K_{-}(\phi, T)$ should be an increasing function of $\phi$ near the fixed point. Hence, a necessary condition for stability of a fixed point solution $\bar{\phi}$ is

$$
\left.\frac{\partial K_{-}(\phi, T)}{\partial \phi}\right|_{\phi=\bar{\phi}}>0 \text {. }
$$

It is simple to establish that $K(\phi, T)$, and hence $K_{-}(\phi, T)$, is $C^{1}$ in the following manner. Denoting ' as differentiation with respect to $\phi$,

$$
K^{\prime}(\phi, T)=-T K(\phi, T)+T\left(1-\mathrm{e}^{-T}\right) \hat{J}(\phi T) .
$$

By construction $K(0, T)=K(1, T)$. Since $g(0)=0$, we also have

$$
\hat{J}(0)=\sum_{j=0}^{\infty} g(j T)=\sum_{j=1}^{\infty} g(j T)=\hat{J}(T)
$$

Hence $K^{\prime}(0, T)=K^{\prime}(1, T)$ and $K(\phi, T)$ is $C^{1}$. However, higher-order derivatives of $K$ have a discontinuity at $\phi=0$. Unfortunately, it is difficult to extend the above stability argument to larger IF networks $(N>2)$, except in special circumstances [32]. Therefore, one must either analyse the spectrum of the linear operator in Eq. (17) or resort to numerical simulations.

Example 2. $(N=4,6)$. As a slightly more complicated example, consider a ring of four oscillators with uniform nearest neighbour coupling $\left(W_{m}=\delta_{m, 1}+\delta_{m, N-1}\right)$ and synaptic delays. The fixed-point spaces for a ring of 

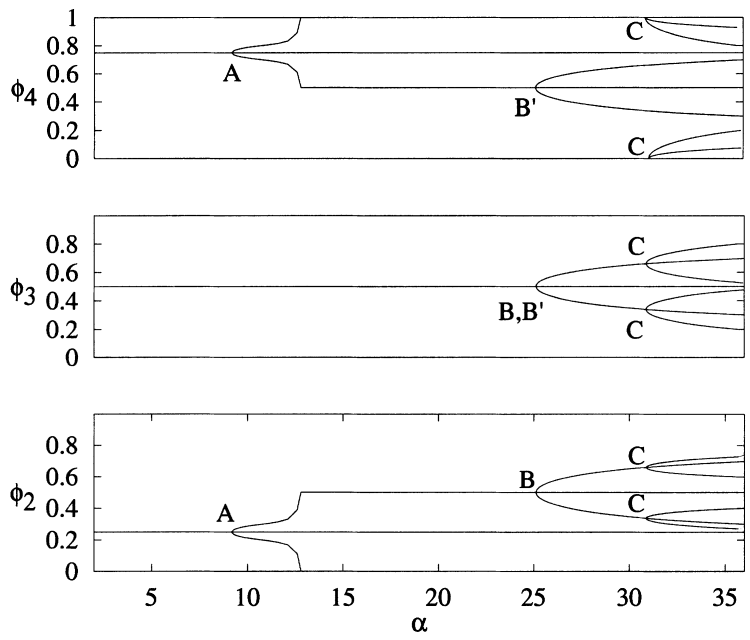

Fig. 3. Relative phase of a ring of four IF oscillators with nearest neighbour coupling and synaptic delays showing bifurcations to isotropy groups with $d>1$ as $\alpha$ is varied $t_{\mathrm{d}}=0.14, I=2$ and $\left.\epsilon=0.05\right)$. Oscillator 1 is taken as the reference oscillator and its phase fixed to zero. At the point A a pair of $d=2$ states of the form $(0, \phi, 1 / 2, \bar{\phi})$ bifurcates from the travelling wave state $\phi_{n}=n / 4$. At the point $B^{\prime}$ a pair of $d=2$ states of the form $(0,0, \phi, \phi)$ bifurcates from the state $(0,0,1 / 2,1 / 2)$ and similarly at point $B$ a pair of the form $(0, \phi, \phi, 0)$ bifurcated from $(0,1 / 2,1 / 2,0)$. At the points $C$, there are bifurcations from $d=2$ states $(0, \phi, \phi, 0)$ to $d=4$ states. The stability of the various branches can be determined numerically. For example, the travelling wave solution is found to be unstable for small $\alpha$ but is stable beyond the bifurcation point $A$.

four oscillators are (from Table 1) as follows: $(\phi, \phi, \phi, \phi),(\phi, \bar{\phi}, \phi, \bar{\phi}),(\phi, \phi, \bar{\phi}, \bar{\phi}),(\phi, \phi+1 / 4, \bar{\phi}, \bar{\phi}+1 / 4)$ for $d=1,\left(\phi_{1}, \phi_{2}, \phi_{1}, \phi_{2}\right),\left(\phi_{1}, \phi_{2}, \overline{\phi_{1}}, \overline{\phi_{2}}\right),\left(\phi_{1}, \phi_{2}, \phi_{2}, \phi_{1}\right),\left(\phi_{1}, \phi_{2}, \overline{\phi_{2}}, \overline{\phi_{1}}\right)$ for $d=2,\left(\phi_{1}, \phi_{2}, \phi_{3}, \phi_{2}\right)$ for $d=3$ and $\left(\phi_{1}, \phi_{2}, \phi_{3}, \phi_{4}\right)$ for $d=4$. In Fig. 3 we illustrate how certain periodic solutions with $d>1$ bifurcate from maximally symmetric solutions as the parameter $\alpha$ is varied for some fixed $\tau_{\mathrm{d}}$. To illustrate the effects of spacedependent delays consider a ring of oscillators with communication delays $\tau_{m}=m \tau_{\mathrm{d}}, P(\tau)=g(\tau)$. Using the distribution (23) in conjunction with Eqs. (4) leads to the $N$ equations:

$$
1=\left(1-\mathrm{e}^{-T}\right) I+\epsilon \sum_{m=1}^{N} W_{m} K\left(\phi_{n+m}-\phi_{n}-m \tau_{\mathrm{d}} / T, T\right), \quad n=1, \ldots, N,
$$

where $K(\phi, T)$ is given by Eq. (31). An example of the so-called in-out phase solution (only possible in even numbered rings) is shown in Fig. 4 for nearest and next-nearest neighbour interactions $\left(W_{m}=1\right.$ if $d_{m} \leq 2$ and zero otherwise). We trace the bifurcation of $(0,1 / 2,0,1 / 2,0,1 / 2)(d=1)$ to $(0, \phi, 0, \phi, 0, \phi)(d=2)$ governed by Eqs. (36) for $N=6$.

Example 3. $(N=3)$. In Fig. 5 we present a numerical construction of the map of inter-spike intervals in the form of a plot of $D(k)$ vs. $D(k-1)$ where $D(k)=T_{k+1}^{1}-T_{k}^{1}$ for a ring of three coupled IF oscillators. (For three oscillators, $\mathbf{D}_{3} \cong \mathbf{S}_{3}$.) Points on the graph are obtained from a direct integration of the IF dynamics (1) and establishing the time of threshold crossings. The points lie on an invariant circle indicating quasiperiodicity (or possibly high order periodicity) as predicted by the linearised theory presented in Section 2.2. It is useful to project the IF state variables from $[0,1]^{N}$ to $\mathbb{C}$ so that trajectories in the space of state variables $U_{m}(t)$ may be visualised. With this in mind we introduce $v_{m}, V(t) \in \mathbb{C}$, where $v_{m}=\exp (2 \pi \mathrm{i} m / N)$ and $V(t)=\sum_{m=1}^{N} U_{m}(t) v_{m}$. In Fig 6 we plot the projected dynamics $V(t)=V_{x}(t)+i V_{y}(t)$ for a range of $\alpha$ values and $N=3$. For three oscillators we have $V_{x}(t)=-(1 / 2)\left(U_{1}(t)+U_{2}(t)-2 U_{3}(t)\right)$ and $V_{y}(t)=\sqrt{3} / 2\left(U_{1}(t)-U_{2}(t)\right)$. The synchronous state is located at the origin $V_{x}=V_{y}=0$, whereas the straight lines $V_{y}=0, V_{y}= \pm V_{x} / \sqrt{3}$ correspond to the two-in-phase states in which two oscillators fire together. The discontinuous nature of the oscillator state variables $U_{m}(t)$ precludes the 


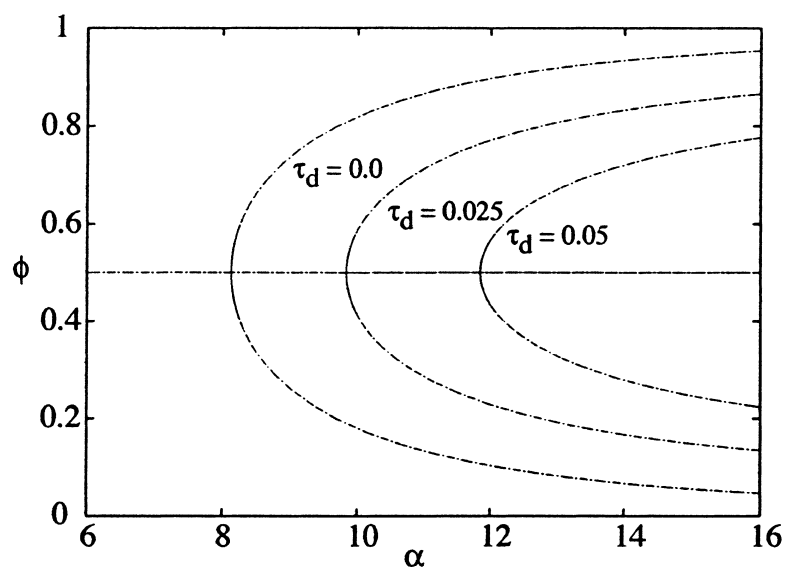

Fig. 4. Effect of space-dependent axonal delays in a ring of six IF oscillators with $I=2$ and $\epsilon=0.01$ and nearest neighbour/next-nearest neighbour coupling. We show solutions bifurcating from $(0,1 / 2,0,1 / 2,0,1 / 2)$ to $(0, \phi, 0, \phi, 0, \phi)$ for varying fundamental units of delay $\tau_{m}=m \tau_{\mathrm{d}}$. All solutions are unstable for $\epsilon>0$, whereas the solution $\phi=1 / 2$ becomes stable beyond the bifurcation point $(\alpha$ increasing) when $\epsilon<0$.

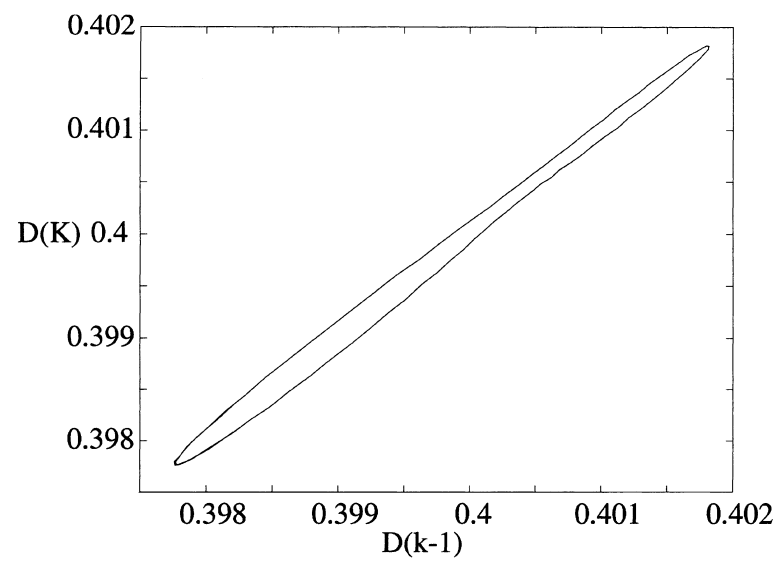

Fig. 5. Inter-spike interval $D(k)$ plotted against the inter-spike interval $D(k-1)$ in a network of three IF oscillators with synaptic coupling. $\tau_{\mathrm{d}}=0, I=2, \alpha=17$ and $\epsilon=0.2$.

possiblility of closed continuous trajectories in the complex plane. One finds that the trajectories consist of three disconnected parts each of which is bounded within a triangular cell as shown in Fig. 6. This reflects an underlying $\mathbf{Z}_{3}$ symmetry. The system jumps discontinuously between these disconnected parts whenever one of the oscillators fire. (Note that the two-in-phase states are invariant under the dynamics since Eq. (1) is first order in time. Thus a trajectory cannot cross the two-in-phase state manifolds smoothly.) For sufficiently small $\alpha$, travelling waves are stable and the corresponding trajectory within a single triangular cell forms a smooth curve in a neighbourhood of the centre of the cell. This is shown in the inset of Fig. 6. The associated inter-spike interval is a constant. For $\alpha$ increasing, a point is reached where the inter-spike interval bifurcates from a stable fixed point to dynamics on an invariant circle (as in Fig. 5). In this case the variation in inter-spike intervals adds another level of structure to the projected dynamics $V(t)$ as illustrated in Fig 6 for $\alpha=17$ and $\alpha=20$. For sufficiently large values of $\alpha$, one finds that the periodic trajectory for $V(t)$ has been destroyed in a global heteroclinic bifurcation (by collision with the borders of the triangular regions). This global bifurcation is analogous to the so-called transcritical/homoclinic bifurcation previously investigated for smoothly coupled oscillators with $\mathbf{S}_{3}$ symmetry (see [23] and Section 4). For solutions bifurcating from the travelling wave with the opposite orientation, the dynamics is similar but occupies 


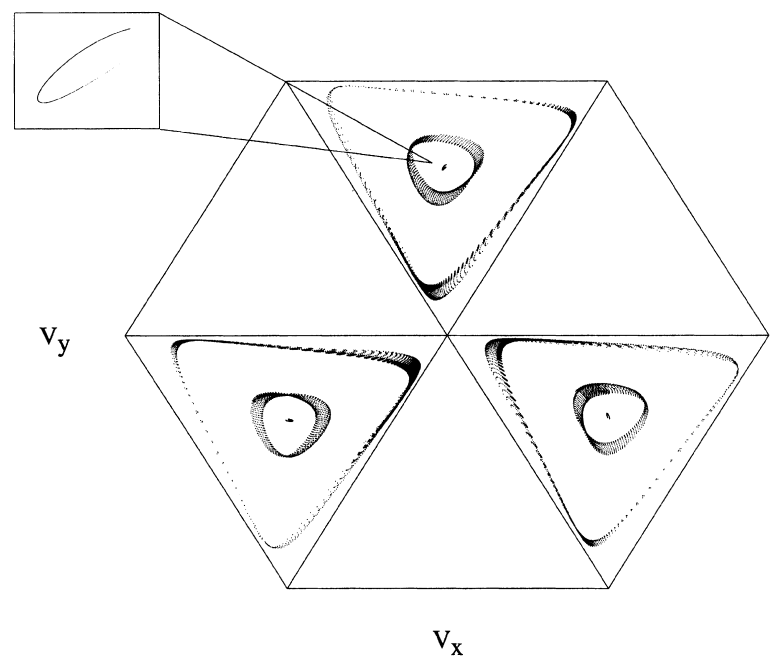

Fig. 6. Projected dynamics for a ring of three IF oscillators with synaptic delays, $\tau_{\mathrm{d}}=0, I=2$ and $\epsilon=0.2$. Here $V(t)=V_{x}(t)+\mathrm{i} V_{y}(t), V_{x}(t)=-(1 / 2)\left(U_{1}(t)+U_{2}(t)-2 U_{3}(t)\right), V_{y}(t)=(\sqrt{3} / 2)\left(U_{1}(t)-U_{2}(t)\right)$. A stable travelling wave $($ seen near the centre of triangular cells) at $\alpha=14$ undergoes a bifurcation to an attractor (shown at $\alpha=17$ ) with structure induced by variation of the inter-spike interval. This attractor approaches the borders of a triangular region with increasing $\alpha$ as seen for $\alpha=20$. A global heteroclinic bifurcation occurs when the attractor collides with the triangular border and the network jumps to a state of near synchrony.

the empty set of triangular regions shown in Fig. 6. It would seem that the above global bifurcation can prevent any possible period-doubling routes to chaos.

\section{Phase-coupled model}

\subsection{Method of averaging for the integrate-and-fire model}

Suppose that in the absence of any coupling, $\epsilon=0$, each oscillator fires with the same period $T_{0}$ where $T_{0}=$ $\int_{0}^{1} \mathrm{~d} U / f(U)$ and we no longer restrict $f$ to be linear. Following [39], we introduce the nonlinear transform $U_{n}(t) \rightarrow$ $\psi_{n}(t)$ according to

$$
(\bmod 1) \psi_{n}(t)+\frac{t}{T_{0}} \equiv \Psi\left(U_{n}(t)\right)=\frac{1}{T_{0}} \int_{0}^{U_{n}(t)} \frac{\mathrm{d} U}{f(U)} .
$$

Under such a transformation Eqs. (1) become

$$
\frac{\mathrm{d} \psi_{n}(t)}{\mathrm{d} t}=\epsilon F\left(\psi_{n}+t / T_{0}\right) \sum_{m=1}^{N} \int_{0}^{\infty} J_{m}(\tau) E_{n+m}(t-\tau) \mathrm{d} \tau,
$$

where

$$
F(z)=\frac{1}{T_{0}} \frac{1}{\left[f \circ \Psi^{-1}(z)\right]}, \quad F(z+j)=F(z), \quad j \in \mathbb{Z} .
$$

The function $F$ may be interpreted as the instantaneous phase-coupling response function of the system. When $\epsilon=0$, the phase variable $\psi_{n}(t)=\psi_{n}$ is constant in time and all oscillators fire with period $T_{0}$. Hence, there is an attracting $N$-torus foliated with periodic orbits of period $T_{0}$. The assumption of strong contraction (compared 
to the strength of coupling $\epsilon$ ) in the neighbourhood of the limit cycles enables one to use normal hyperbolicity (see [16] for a discussion) to predict persistence of an $N$-torus which is asymptotically attracting when $\epsilon$ is small. If in the presence of coupling the right-hand side of (38) is periodic one may invoke the averaging theorem [12] to obtain a first order normal form for the asymptotic dynamics of equations (38). One might suppose, to a first approximation, that for weak coupling ( $\epsilon$ small) each oscillator still fires with period $T_{0}$ but now the phases $\psi_{n}(t)$ slowly drift according to Eq. (38). By assumption, the delay distribution $J_{m}(\tau)$ is normalisable $\left(\int_{0}^{\infty} J_{m}(\tau) \mathrm{d} \tau<\infty\right)$ with $J_{m}(\tau) \rightarrow 0$ as $\tau \rightarrow \infty$. Hence, we can neglect the contributions to $E_{m}(t)$ from firing-events sufficiently far in the past such that, to first-order in $\epsilon$, the firing-times may be approximated by $T_{j}^{n}=\left(j-\psi_{n}(t)\right) T_{0}$. The right-hand side of Eq. (38) then becomes a $T_{0}$ periodic function of $t$, thus satisfying the conditions for the averaging theorem to apply. Introducing the autonomous phase interaction function:

$$
H_{m}(\psi)=\frac{1}{T_{0}} \int_{0}^{\infty} J_{m}(\tau) F\left[\tau / T_{0}-\psi\right] \mathrm{d} \tau
$$

allows us to state the averaging theorem in the following manner. There exists a change of variables, $\psi \rightarrow \psi+$ $\epsilon w(\psi, t, \epsilon)$ that maps solutions of (38) to those of

$$
\frac{\mathrm{d} \psi_{n}}{\mathrm{~d} t}=\epsilon \sum_{m=1}^{N} H_{m}\left(\psi_{n+m}-\psi_{n}\right)+\mathrm{O}\left(\epsilon^{2}\right) .
$$

It may be shown that the function $w(\psi, t, \epsilon)$ is not small when $t \rightarrow \infty$. However, for $\epsilon \ll 1$, the dynamics of (38) are $\epsilon$-close to those of (41) for times of $\mathrm{O}\left(\epsilon^{-1}\right)$. For $\epsilon$ small enough hyperbolic periodic orbits (including fixed points) of (41) correspond to hyperbolic periodic orbits of (38). Periodic orbits of (41) which have two or more zero Floquet exponents may or may not imply a periodic orbit with neutral stability in the unaveraged system (38). Higher-order corrections to (41) can destroy such orbits. Since saddle connections may not exist on the limited timescale in which averaging guarantees shadowing to $\mathrm{O}\left(\epsilon^{-1}\right)$, heteroclinic chaos in (38) may be suppressed by the averaging process. However, saddle connections will persist if the stable and unstable manifolds are contained in $\operatorname{Fix}(\Sigma)$, with $\Sigma$ a subgroup of the full group of symmetries of equations(38).

Eqs. (41) immediately show that the averaging process reduces the dynamics to one of phase-differences only. To $\mathrm{O}(\epsilon)$ the phase interaction function (40) is simply the average of the right-hand side of (38) over a single period. Moreover, delays in the propagation of signals between pulse-coupled oscillators reduce to phase shifts in the corresponding phase-coupled model. The phase interaction may be interpreted in a neural context as follows (after a change of variables $\tau \rightarrow \tau / T_{0}$ in Eq. (40)). The effective interaction between the pre-synaptic neuron labelled at $n$ and the post-synaptic neuron at $n+m$ is obtained by convolving over one period of oscillation the weighted synaptic current $J_{m}\left(\tau T_{0}\right)$ with the response function $F\left(\tau-\left(\psi_{n+m}-\psi_{n}\right)\right)$. For instantaneous coupling between neurons such that post-synaptic currents are unit delta functions of the form $W_{m} \delta(\tau)$, then $H_{m}(\psi) \rightarrow H_{m}^{\infty}(\psi)=W_{m} F(-\psi)$. Hence, if the interaction function for an instantaneous synapse is known, the general phase interaction function can be obtained as a convolution since

$$
H_{m}(\psi)=\frac{1}{T_{0}} \int_{0}^{\infty} J_{m}(\tau) H_{m}^{\infty}\left[\psi-\tau / T_{0}\right] \mathrm{d} \tau .
$$

The function $F(-\psi)$ is sometimes referred to as the phase resetting curve of a neuron. If $F(-\psi)>0$, a small and instantaneous depolarization at the neuronal phase $\psi$ will advance the next firing event. The response of the neuron to excitatory inputs is said to be of type I. The response is said to be of type II if a stimulus can either advance or retard the phase depending upon the time at which it is administered. Integrate-and-fire neurons (with $f(U)=-U+I)$ have a type I response whilst limit cycle oscillators based upon Hodgkin-Huxley like models of excitable cells are of type II. When describing a piece of cortex or a CPG circuit with a set of oscillators the biological realism of the model typically resides in the phase interaction function. The distinction between type I and type II response is unambiguous for networks with either purely excitatory or purely inhibitory coupling as considered in this paper. 
However, patterns of excitation and inhibition in a network of type I oscillators can also lead to responses from individual neurons that resemble those of a type II neuron in isolation. This interesting possibility is explored in [53].

\subsection{Phase-locked solutions}

Following our analysis of the pulse-coupled model, we first consider phase-locked solutions of Eq. $(41), \psi_{n}(t)=$ $\phi_{n}+\Omega t$, where $\phi_{n}$ is a constant phase and $\Omega$ is an $\mathrm{O}(\epsilon)$ contribution to the effective frequency of the oscillators, that is, $1 / T=1 / T_{0}+\Omega$. Substitution into Eq. (41) and working to $\mathrm{O}(\epsilon)$ leads to the fixed point equations:

$$
\Omega=\epsilon \sum_{m=1}^{N} H_{m}\left(\phi_{n+m}-\phi_{n}\right), \quad n=1, \ldots, N .
$$

Eqs. (43) directly correspond to the conditions (4) for phase-locked solutions of the integrate-and-fire model and have the same underlying symmetry group $\mathbf{D}_{N} \times \mathbf{S}^{1}$. Note, however, that phase-locked solutions of Eq. (43) are now independent of $\epsilon$; the strength of coupling only affects the frequency $\Omega$. In order to analyse the local stability of a phase-locked solution satisfying Eqs. (43), we linearise Eq. (41) by setting

$$
\psi_{n}(t)=\phi_{n}+\Omega t+\theta_{n}(t)
$$

and expand to first-order in $\theta_{n}$ to obtain

$$
\frac{\mathrm{d} \theta_{n}}{\mathrm{~d} t}=\sum_{m=1}^{N} \mathcal{H}_{n m}(\Phi)\left[\theta_{m}-\theta_{n}\right]
$$

where $\mathcal{H}_{n m}(\Phi)=\epsilon H_{m-n}^{\prime}\left(\phi_{m}-\phi_{n}\right)$. The Floquet exponents of a periodic orbit are simply given by the eigenvalues of the Jacobian matrix $\hat{\mathcal{H}}_{n m}(\Phi)=\mathcal{H}_{n m}(\Phi)-\delta_{n m} \sum_{k=1}^{N} \mathcal{H}_{n k}(\Phi)$. One of these eigenvalues is always zero, and the corresponding eigenvector points in the direction of the flow, that is, $(1,1, \ldots, 1)$. The phase-locked solution will be stable provided that all other eigenvalues have a negative real part. Phase-locked solutions of the phasecoupled model can bifurcate whenever there exists more than one eigenvalue with zero real part (non-hyperbolic solutions). If one or more real eigenvalues cross the imaginary axis, then the bifurcating branches correspond to other phase-locked solutions.

It is also possible for Hopf bifurcations to occur leading to non-phase-locked behaviour. As a simple illustration, we follow Ref. [41] and consider travelling wave solutions of the form $\psi_{n}(t)=n \beta+\Omega t$, where, $\beta=0$ corresponds to a synchronous solution and $\beta=n_{b} / N, n_{b}=1, \ldots, N-1$, corresponds to a travelling wave solution. Substitution into Eq. (45) gives the disperison relation

$$
\Omega=\epsilon \sum_{m=1}^{N} H_{m}(m \beta), \quad n=1, \ldots, N .
$$

The elements $\mathcal{H}_{n m}(\Phi)$ become $\epsilon H_{m-n}^{\prime}((m-n) \beta)$. The fact that $\mathcal{H}_{n m}(\Phi)$ now depends on $m-n(\bmod N)$ means that the eigenvectors of the Jacobian matrix are of the form

$$
\theta_{n}(t)=\mathrm{e}^{\lambda_{p} t+2 \pi \mathrm{i} n p / N}, p=0,1, \ldots, N-1,
$$

and the eigenvalue $\lambda_{p}$ satisfy [41]:

$$
\lambda_{p}=\epsilon \sum_{m=1}^{N} H_{m}^{\prime}(m \beta)\left[\mathrm{e}^{2 \pi \mathrm{i} p m / N}-1\right] .
$$


A travelling wave solution will be stable provided that $\operatorname{Re}\left(\lambda_{p}\right)<0$ for all $p \neq 0$. (As noted previously, the eigenvalue for $p=0$ is neutrally stable.) If $N$ is odd, then $\lambda_{0}$ is real and the rest of the eigenvalues occur in complex conjugate pairs $\lambda_{p}$ and $\lambda_{-p}$. The structure of the eigenvalue [48] (with $p>0$ ) implies that typically the real part of just one pair can be made to vanish for some natural choice of the distribution $J_{m}(\tau)$. This indicates that the generic bifurcation of a travelling wave state, for an odd number of oscillators, is a simple Hopf bifurcation. An example of a supercritical Hopf bifurcation is shown in Fig. 8 (as part of a more complicated bifurcation sequence, see below).

The relationship between phase-locked solutions of the phase-coupled model and the original pulse-coupled model can be clarified in the limit of weak-coupling $(\epsilon \rightarrow 0)$. If we set $f(U)=-U+I$, then

$$
F(\psi)=\frac{\mathrm{e}^{T_{0} \psi}}{I T_{0}}
$$

with $T_{0}=\ln [I /(I-1)]$. Comparison of Eqs. (40) and (49) with Eqs (5) and (6) then shows that the phase-interaction function is proportional to the interaction function of the pulse-coupled model,

$$
H_{m}(\phi)=\frac{\mathrm{e}^{T_{0}} K_{m}\left(\phi, T_{0}\right)}{I T_{0}^{2}} .
$$

Hence Eqs. (4) reduce to Eqs. (43) to first-order in $\epsilon$ and the phase-locked solutions of the IF model converge to those of the phase-coupled model in the limit $\epsilon \rightarrow 0$. This is illustrated for two oscillators in Fig. 2. The conditions for the stability of phase-locked solutions also converge in the weak coupling limit. Eq. (50) implies that the matrix $\mathcal{H}$ of Eq. (45) is proportional to the matrix $\mathcal{K}$ of Eq. (20) and hence $\hat{\mathcal{H}}$ and $\hat{\mathcal{K}}$ have the same eigenvalues. We have now established the following important result: if there exists a stable or unstable (hyperbolic) phase-locked solution of the phase-coupled model, for any finite $N$, then there exists a corresponding solution of the pulse-coupled model of the same stability type for sufficiently small $\epsilon$. This extends to the case of the discontinuous IF model, the well-known result that the existence of hyperbolic periodic solutions in a phase-reduced model implies their existence in the full oscillator model for sufficiently smooth systems.

\section{3. $\mathbf{S}_{3}$ global heteroclinic bifurcation}

As shown in Section 3, numerical integration of a ring of three IF oscillators reveals the existence of a codimension one global bifurcation from a travelling wave to a synchronous state (see Fig. 6.) This type of bifurcation was previously studied by Ashwin and co-workers [23] for a system of three weakly coupled Van der Pol oscillators with $\mathbf{S}_{3}$ symmetry. They showed both theoretically, using averaging theory, and experimentally that a transition from a travelling wave to a synchronous state may occur via a homoclinic bifurcation as some system parameter is varied. This typically happens in the following manner: (a) a travelling wave is the only stable solution, (b) there is a supercritical Hopf bifurcation, (c) the only stable solution is a limit cycle, and (d) the cycle grows until it is destroyed at an $\mathbf{S}_{3}$ transcritical/homoclinic bifurcation which stabilises the synchronous solution. (Extensions of this form of global bifurcation to networks with $\mathbf{S}_{N}$ symmetry $(N>3)$ are discussed in [21].) We shall now investigate more closely the global bifurcation of an IF network using the weakly coupled phase model given by Eq. (41). It will turn out that it differs in structure to the homoclinic bifurcation observed by Ashwin et al. [23]. For the sake of illustration, we consider phase-coupled oscillators satisfying Eq. (41) to $\mathrm{O}(\epsilon)$ with $H$ given by Eqs. (40) and (49) and $K_{m}\left(\phi, T_{0}\right)$ satisfying Eq. (5). We also take $T_{0}=\ln 2$ and set $J_{m}(\tau)=g(\tau), m=1,2,3$, where $g(\tau)$ is the $\alpha$ function (24). In Fig. 7 we follow the relative phase between oscillators as a function of the inverse rise time $\alpha$. For small $\alpha$ the travelling wave state is stable. However, with increasing $\alpha$ this solution loses stability via a supercritical Hopf bifurcation at $\alpha=\alpha_{\mathrm{H}} \approx 8$ and a stable limit cycle is created. The relative phases $\hat{\psi}_{2}(t)=\psi_{2}(t)-\psi_{1}(t)$ and $\hat{\psi}_{3}(t)=\psi_{3}(t)-\psi_{1}(t)$ are related via $\hat{\psi}(t)=1-\hat{\psi}_{3}(t+\hat{T} / 3)$, where $\hat{T}$ is the common period of the limit cycle. The relative shift in time by $\hat{T} / 3$ reflects an additional $\mathbf{S}^{1}$ symmetry associated with time-shifts of periodic solutions around the limit cycle and is an example of a simple Hopf bifurcation with symmetry (see [22]). Until $\alpha$ reaches some critical point, $\alpha=\alpha_{\mathrm{c}}$, the amplitude of oscillation continues to grow. However, at $\alpha_{\mathrm{c}}$, the limit 


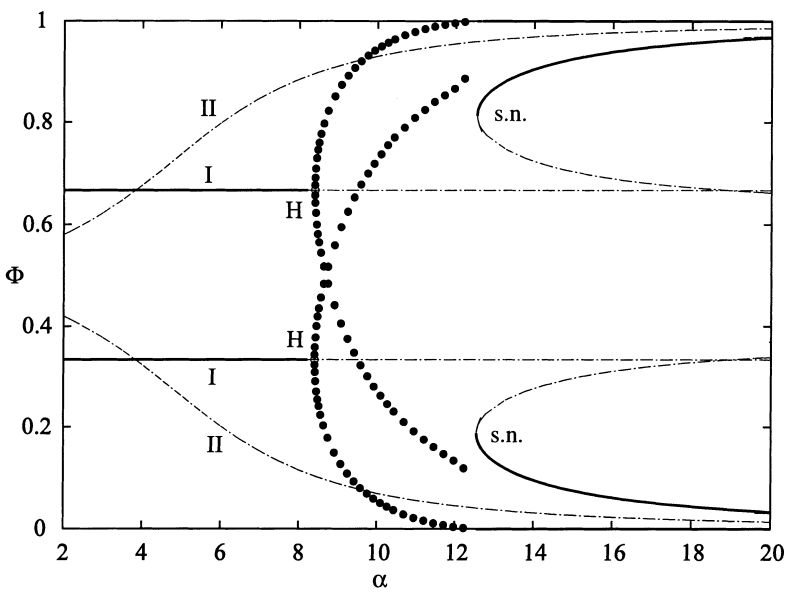

Fig. 7. An illustration of the $\mathbf{S}_{3}$ heteroclinic bifurcation for 3 phase-coupled oscillators. The travelling wave state $\left(\Phi=\left(0, \hat{\psi}_{2}, \hat{\psi}_{3}\right)=\right.$ $(0,1 / 3,2 / 3))$ indicated by $\mathrm{I}$ is stable for low $\alpha$ (solid lines) and loses stability (dashed lines) due to a supercritical Hopf bifurcation $(\mathrm{H})$ at $\alpha=\alpha_{\mathrm{H}} \approx 8$. Filled circles denote the amplitude of the stable limit cycle of the relative phases $\hat{\psi}_{2}(t)$ and $\hat{\psi}_{3}(t)$. The merger of the limit cycle and the 2-in-phase invariant manifold at $\alpha=\alpha_{\mathrm{c}} \approx 12$ destroys the limit cycle and leads to the creation of stable/unstable pairs of 2-in-phase states via a saddle node bifurcation (s.n.). There exist additional unstable 2-in-phase solutions indicated by II.

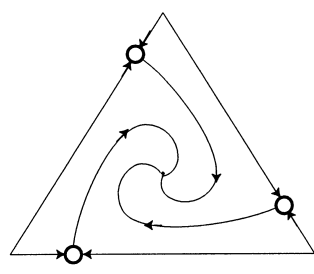

(a) $\alpha<\alpha_{H}$

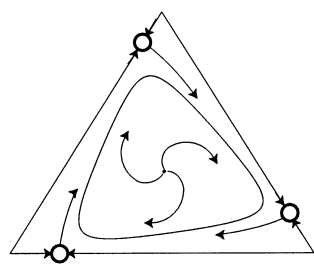

(b) $\alpha_{\mathrm{H}}<\alpha<\alpha_{\mathrm{c}}$

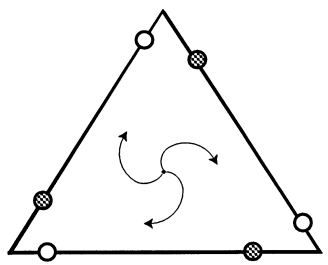

(c) $\alpha=\alpha_{c}$

Fig. 8. An alternative illustration of the $\mathbf{S}_{3}$ transcritical/homoclinic bifurcation in the projected coords $V_{x}(t)=-(1 / 2)\left(\psi_{1}(t)+\psi_{2}(t)-2 \psi_{3}(t)\right)$, $V_{y}(t)=(\sqrt{3} / 2)\left(\psi_{1}(t)-\psi_{2}(t)\right)$. The travelling wave state is represented by the point in the centre of the triangular cell. The (unstable) synchronous state occupies the vertices of the triangle, whilst the 2-in-phase states arise on the borders of the triangle. As $\alpha$ increases the travelling wave loses stability $\left(\alpha=\alpha_{\mathrm{H}}\right)$ and a stable limit cycle emerges with amplitudes that increase with $\alpha$ until colliding with the border of the triangle $\left(\alpha=\alpha_{\mathrm{c}}\right.$ ), where it is destroyed in a heteroclinic bifurcation. Coincident with this heteroclinic bifurcation is the creation of stable/unstable pairs of 2-in-phase states. The limit cycle shown in (b) is obtained numerically for $\alpha=10$.

cycle is destroyed in a global heteroclinic bifurcation (due to collision with invariant manifolds associated with the 2-in-phase solutions). Simultaneous with the heteroclinic bifurcation at $\alpha_{\mathrm{c}}$ is a saddle-node bifurcation in which stable/unstable pairs of 2-in-phase solutions are created. There are additional unstable 2-in-phase states that exist for all $\alpha$, together with an unstable synchronous solution. In Fig. 8 we illustrate the global bifurcation schematically along similar lines to [23] by considering a projection of the absolute phase variables to the complex plane with $V(t)=\sum_{m=1}^{3} \psi_{m}(t) \mathrm{e}^{2 \pi \mathrm{i} m / 3}$. For clarity only one dynamically invariant triangle of the lattice is shown. Fixed points of the dynamics are the travelling wave state (centre of the triangle), the synchronous state (triangle vertices) and the two-in-phase states (located at a point on each edge of the triangle). The bifurcation sequence with increasing $\alpha$ is shown in Figs. 8(a)-(c) with the limit cycle in (b) obtained numerically for $\alpha=10$.

\subsection{Comparison with Kuramoto model}

It is interesting to contrast the behaviour of the phase-coupled model [41] having a type I response function derived from the IF model of Section 2 (see Eq. (49)), with one having a type II response function given 
by a sinusoid

$$
F(\psi)=-\sin (2 \pi \psi) .
$$

In the latter case, Eq. (41) reduces to the well-known Kuramoto model with distributed phase-shifts

$$
\frac{\mathrm{d} \psi_{n}}{\mathrm{~d} t}=\frac{1}{T_{0}} \sum_{m} \int_{0}^{\infty} J_{m}(\tau) \sin \left[2 \pi\left(\psi_{n+m}-\psi_{n}\right)-\omega \tau\right] \mathrm{d} \tau .
$$

A similar equation arises in the analysis of the dissipative, overdamped resistively loaded Josephson array [4,18]. For concreteness, suppose that the distribution $J_{m}(\tau)$ has the product form $J_{m}(\tau)=W_{m} P(\tau)$ with the weight $W_{m}$ assumed to be (a) symmetric, $W_{m}=W_{N-m}$, and (b) a monotonically decreasing function of the separation on the ring $d_{m}$ (see Section 2.3). As in our previous analysis, we can exploit the underlying $\mathbf{D}_{N} \times \mathbf{S}^{1}$ symmetry of Eq. (52) to investigate phase-locked solutions. In particular, maximally symmetric solutions such as travelling waves are an immediate consequence of this symmetry.

In order to indicate some of the special features of the Kuramoto model, we shall analyse the stability of travelling wave solutions. First, it is useful to introduce the following Fourier transforms:

$$
\begin{aligned}
& \Lambda(p)=\sum_{m=1}^{N} W_{m} \cos (2 \pi m p / N), \\
& \Delta(\omega)=\frac{1}{T_{0}} \int_{0}^{\infty} P(\tau) \exp (\mathrm{i} \omega \tau) \mathrm{d} \tau,
\end{aligned}
$$

and set $\Delta_{\mathrm{c}}(\omega)=\operatorname{Re} \Delta(\omega), \Delta_{S}(\omega)=\operatorname{Im} \Delta(\omega)$. Substituting Eq. (51) into (40) and exploiting the symmetry of the interaction function $W_{m}$, one finds that the dispersion relation (46) becomes

$$
\Omega(\beta, \omega)=-\Delta_{s}(\omega) \Lambda(\beta)
$$

Similarly, linearising Eq. (52) about a travelling wave state leads to a Jacobian with eigenvalues given by Eq. (48), which on using Eq. (51) and equating real and imaginary parts gives

$$
\begin{aligned}
& \operatorname{Re} \lambda_{p}=\pi \Delta_{c}(\omega)[\Lambda(p+\beta)+\Lambda(p-\beta)-2 \Lambda(\beta)], \\
& \operatorname{Im} \lambda_{p}=\pi \Delta_{s}(\omega)[\Lambda(p-\beta)-\Lambda(p+\beta)] .
\end{aligned}
$$

Note that Eqs. (55)-(57) exhibit a simple product form in relation to the dependence on (a) the spatial structure of the connections specified by $W_{m}$, and (b) the distribution of delays $P(\tau)$. This special feature of the Kuramoto model leads to non-generic behaviour as will be described below.

We first consider the stability of the synchronous state $\beta=0$ for which $\lambda_{p}$ is real for all $p$. Since $W_{m}$ decreases monotonically with $d_{m}$ it follows that $\max _{p} \Lambda(p)=\Lambda(0)$, and hence that the synchronous state is stable (unstable) if $\Delta_{\mathrm{c}}(\omega)>0\left(\Delta_{\mathrm{c}}(\omega)<0\right)$. The condition $\Delta_{\mathrm{c}}(\omega)=0$ determines a degenerate bifurcation point where $\lambda_{p}=0$ for all $p$. We now calculate $\Delta_{\mathrm{c}}(\omega)$ for each of the three sources of delay listed in Section 2.3.

Uniform axonal delay. Assume that each axonal connection has the same transmission delay $\tau_{\mathrm{d}}$ so that $P(\tau)=$ $\delta\left(\tau-\tau_{\mathrm{d}}\right)$ and $\Delta_{\mathrm{c}}(\omega)=T_{0}^{-1} \cos \left(\omega \tau_{\mathrm{d}}\right)$. It follows that for excitatory coupling the synchronous state is stable for sufficiently small delays, $\tau_{\mathrm{d}}<T_{0} / 2$. As $\tau_{\mathrm{d}}$ increases alternating bands of stability and instability are generated. (The effects of space-dependent axonal delays are considered by Crook et al. [45]. They show that destabilisation of the synchronous state due to an increase in delays can lead to travelling waves. They also suggest that this could account for the fact that oscillatory behaviour in the visual cortex tends towards synchrony, whereas the olfactory cortex tends to produce travelling oscillatory waves; the latter has long-range excitatory connections and hence longer axonal delays. An alternative mechanism base on dendritic structure is presented in [46].) 
Synaptic processing. Take $P(\tau)$ to be the $\alpha$-function (24). Substituting into Eq. (54) and performing the integration over $\tau$ gives

$$
\Delta(\omega)=\frac{1}{T_{0}} \frac{\alpha^{2}\left(\alpha^{2}-\omega^{2}+2 \mathrm{i} \alpha \omega\right)}{\left(\alpha^{2}+\omega^{2}\right)^{2}} .
$$

Hence, for excitatory coupling the synchronous state is stable if $\alpha>\omega$ (fast synapse) and unstable if $\alpha<\omega$ (slow synapse). The desynchronising effects of synapses was previously highlighted by van Vreeswijk et al. [39] in their slow study of two pulse-coupled oscillators, and this theme has been further developed elsewhere [20,51,52].

Dendritic processing. Suppose that there exists a distribution of axo-dendritic connections with some spatially periodic component of the form

$$
W_{m}(\xi)=W_{m} \cos \left(p \xi+\xi_{0}\right),
$$

where $\xi_{0}$ represents an offset of the spatially periodic stimulation of frequency $p$. Making use of the following Fourier representation of the fundamental solution $G(\xi, t)$ :

$$
G(\xi, \tau)=\int_{-\infty}^{\infty} \exp [\mathrm{i} k \xi-\nu(k) \tau] \frac{\mathrm{d} k}{2 \pi}, \quad \nu(k)=D k^{2}+\tau_{s}^{-1},
$$

and substituting Eq. (59) into (29) shows that

$$
P(\tau)=\mathrm{e}^{-v(p) \tau} \cos \left(\xi_{0}\right) .
$$

Substituting Eq. (61) into (54) and performing the integration over $\tau$ gives

$$
\Delta(\omega)=-\frac{1}{T_{0}}\left[\frac{v(p)+\mathrm{i} \omega}{v(p)^{2}+\omega^{2}}\right] \cos \left(\xi_{0}\right) .
$$

Since $v(p)>0$ for all $p$ the synchronous state is stable $(\epsilon>0)$ if $\pi / 2<\xi_{0}-2 k \pi<3 \pi / 2$ with $k \in \mathbb{Z}$.

In order to determine the stability of travelling wave solutions $(\beta \neq 0)$, it is necessary to specify the form of the interaction function $W_{m}$. For the sake of illustration, we shall consider the particular example of a step-function

$$
W_{m}=\Theta\left(L-d_{m}\right), \quad L>[N / 2] .
$$

The parameter $L$ determines the range of interactions. For this choice of $W_{m}$, the function $\Lambda(p)$ of Eq. (53) becomes

$$
\Lambda(p)=\frac{\sin (2 L+1) \pi p}{\sin \pi p} .
$$

We also set

$$
\hat{\Lambda}(p, \beta)=\Lambda(p+\beta)+\Lambda(p-\beta)-2 \Lambda(\beta) .
$$

There are then two conditions under which a travelling wave can be stable: either (I) $\hat{\Lambda}(p, \beta)<0$ for all $p \neq 0$ and $\Delta_{\mathrm{c}}(\omega)>0$ or (II) $\hat{\Lambda}(p, \beta)>0$ for all $p \neq 0$ and $\Delta_{\mathrm{c}}(\omega)<0$. The stability results for the step interaction functions are shown in Fig. 9 for a range of values of the interaction length $L, 0 \leq L \leq N / 4$ and $N=101$. For each $L$, the wave number $\beta$ that satisfy stability condition (I) or (II) are indicated. We see that there are two stability bands. The first (labelled by $\diamond$ 's) consists of travelling wave states that are stable when $\Delta_{\mathrm{c}}(\omega)>0$. This band becomes thinner as $L$ increases from zero until only the synchronous state remains stable. Note that the so-called splay state $(\beta=1)$, where the phases are uniformly spaced around the circle, maintains stability for the largest value of $L$. The second band (labelled by +'s) represents travelling wave states that are stable when $\Delta_{\mathrm{c}}(\omega)<0$, that is, when the synchronous state is unstable. We conclude that there are two mechanisms whereby a synchronous state can be destabilised: (a) if $\Delta_{\mathrm{c}}(\omega)>0$, then a sufficiently large perturbation is needed to induce a transition into the basin of 


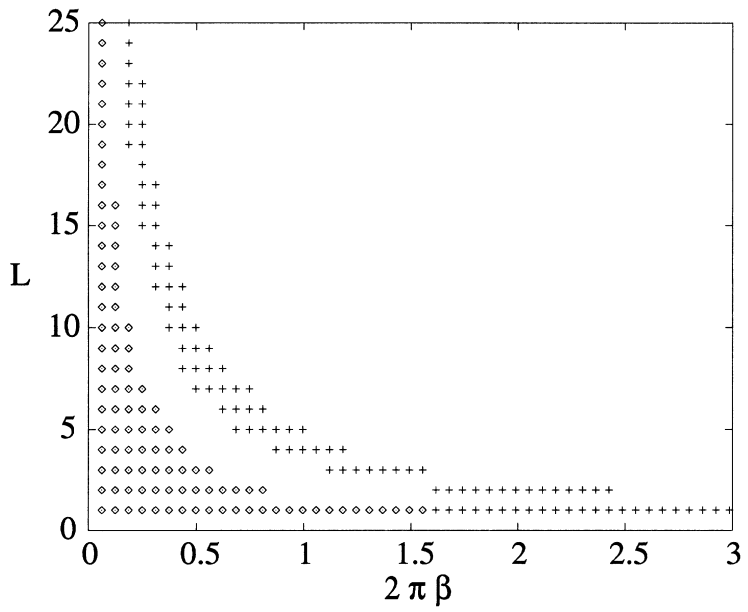

Fig. 9. $W_{m}=\Theta\left(L-d_{m}\right), L<$ [N/4]. Stability of travelling wave solutions with wave number $\beta$ as a function of the interaction length $L$. Diamonds (+'s) indicate states that are stable when $\Delta_{\mathrm{c}}(\omega)>0\left(\Delta_{\mathrm{c}}(\omega)<0\right)$.

attraction of a stable travelling wave satisfying (I), (b) $\Delta_{\mathrm{c}}(\omega)$ becomes negative leading to the formation of a stable travelling wave satisfying (II). The picture for $L \leq N / 4$ should be contrasted with the case of all-to-all coupling $(L=[N / 2])$, where $\Lambda(p)=N \delta_{p, 0}$ so that $\hat{\Lambda}(p, \beta)=N\left[\delta_{p, \beta}+\delta_{p, 1-\beta}\right]$ for $\beta \neq 0$ and $\hat{\Lambda}(p, 0)=-2 N$ for all $p \neq 0$. One then finds that all travelling wave solutions are stable $\left(\Delta_{\mathrm{c}}(\omega)<0\right)$ or unstable $\left(\Delta_{\mathrm{c}}(\omega)>0\right)$ in two eigen directions and marginally stable in the remaining $N-2$ eigen directions. This basic result extends to more general solutions as shown by Watanabe and Strogatz [4].

The above analysis highlights a number of important features of the Kuramoto model that are not typical of more general choices of the phase interaction function such as Eq. (49). First, decoupling of the dynamical system (52) can occur if either $\Lambda(\beta)=0$ or $\Delta_{s}(\omega)=0$. In this case, instead of travelling waves, there can exist invariant $N$-tori in the phase space foliated with limit cycles of equal period. On these foliated tori the flow factorises into a product of flows giving the appearance of $N$ uncoupled oscillators. For example, a nearest-neighbour interaction of the form $W_{m}=\delta_{m, 1}+\delta_{m, N-1}$ causes decoupling of oscillators when $\beta=1 / 4,3 / 4$. Decoupled solutions cannot be continued in system parameters. A further discussion of decoupling can be found in [21]. Second, in the case of an odd numbered ring and $\Delta_{\mathrm{c}}(\omega)=0$ we have $\operatorname{Re} \lambda_{p}=0$ for all $p, \beta$ so that $(N-1) / 2$ pairs of complex conjugate roots $\left(\lambda_{p}\right.$ and $\left.\lambda_{-p}\right)$ cross the imaginary axis simultaneously. Thus, in the special case of the Kuramoto model, the underlying symmetry has forced multiple eigenvalues and the standard Hopf theorem is no longer applicable. The eigenspaces associated with these eigenvalues are often termed modes. Modes that become unstable simultaneously (as their corresponding eigenvalues cross the imaginary axis) may interact nonlinearly to create more complicated behaviour than that which might be expected from them individually. The nature of these mode interactions will not be pursued here.

An even more striking property of the Kuramoto model occurs in the case of global coupling $\left(W_{m}=1 / N\right.$ for all $m=1, \ldots, N)$. As established by Watanabe and Strogatz [4], the system is completely integrable when $\Delta_{\mathrm{c}}(\omega)=0$. Their analysis is based on the observation that each trajectory of the system is actually confined to a three-dimensional subspace $(\Theta(t), \Psi(t), \gamma(t))$. This follows from the change of variables

$$
\tan \left[\pi\left(\psi_{n}(t)-\Theta(t)\right)\right]=\sqrt{\frac{1+\gamma(t)}{1-\gamma(t)}} \tan \left[\pi\left(\phi_{n}-\Psi(t)\right)\right],
$$

where the $\phi_{n}$ are constants and $0 \leq \gamma(t)<1$. It can further be shown that $\Theta(t)$ is passively driven by $\gamma(t)$, $\Psi(t)$ and the dynamics of the latter two variables is characterised by the existence of a Lyapunov function $\mathcal{E}$ such that

$$
\mathrm{d} \mathcal{E} / \mathrm{d} t=R^{2}(t) \Delta_{\mathrm{c}}(\omega)
$$


where $R(t)$ is an order parameter that measures the degree of coherence of the system,

$$
R(t) \exp [2 \pi \mathrm{i} \bar{\psi}(t)]=\frac{1}{N} \sum_{n=1}^{N} \exp \left[2 \pi \mathrm{i} \psi_{n}(t)\right] .
$$

(Note that Watanabe and Strogatz [4] only considered a single phase-shift $\tau$ for which $\Delta_{\mathrm{c}}(\omega)=\cos (\omega \tau)$. However, their analysis carries over to the case of distributed phase-shifts on using our more general expression for $\Delta_{\mathfrak{c}}(\omega)$.) The following results thus hold for almost all initial conditions:

(i) If $\Delta_{\mathfrak{c}}(\omega)>0$, then $\mathcal{E}(t) \rightarrow \infty, \gamma(t), R(t) \rightarrow 1$, and the system converges to the synchronous state.

(ii) If $\Delta_{\mathrm{c}}(\omega)<0$, then $\mathcal{E}(t), \gamma(t), R(t) \rightarrow 0$, and the system converges to an $(N-3)$-dimensional manifold of incoherent states. Such a manifold consists of contant states $\psi_{n}$ satisfying $\sum_{n} \exp \left[2 \pi \mathrm{i} \psi_{n}\right]=0$. There are $(N-2)$ neutrally stable directions around each such incoherent state.

(iii) If $\Delta_{\mathrm{c}}(\omega)=0$, then the system is completely integrable with $\mathcal{E}$, a conserved quantity. Trajectories run along the contours of $\mathcal{E}$ and correspond to periodic motion in $(\Psi, \gamma)$ space. In the full phase space, the motion is quasiperiodic on 2-tori.

We conclude that in contrast to the time-averaged IF model, travelling wave states in a globally coupled Kuramoto network do not destabilise to form limit cycle oscillations via a Hopf bifurcation. This is due to the integrability of the system when $\Delta_{\mathrm{c}}(\omega)=0$. Finally, note that the high degree of marginal stability found in the Kuramoto model is destroyed by including higher harmonics in the response function $F(\psi)$ of Eq. (49). This is discussed in some detail for globally coupled networks by Golomb et al. [54].

\section{Conclusion}

In this paper we have developed a systematic approach to analysing systems of pulse-coupled oscillators with periodic boundary conditions and spatio-temporal symmetries. The identification of maximally symmetric states combined with numerical continuation and bifurcation detection has shown that spontaneous symmetry breaking can play a practical role in the construction of other symmetric solutions. Such a tactic may also prove successful in analysing systems of phase-locked loops in which the frequency (rather than the phase) of an oscillator is updated discontinuously [55]. Our attention has focused upon phase-locked solutions of the integrate-and-fire oscillator for which it has been possible to construct a criterion for linear stability. A natural way to establish the stability of solutions is also to examine a reduced model obtained via averaging. For the first time we have translated statements regarding stability of the pulse-coupled system to those of a corresponding phase-coupled model. The analysis of non-phase-locked states has also been explored by (i) direct numerical integration of the equations of motion showing the variation of the inter-spike interval and (ii) by detecting Hopf bifurcation points in the phase-model and numerically continuing to periodic limit cycles. Numerical results suggest that global bifurcations in the IF system have counterparts in the corresponding phase-model. Moreover, we have constructed phase portraits of the IF and phase-reduced system showing that symmetries of the underlying dynamics are inherited by attractors. Interestingly, the symmetry of a forced coupled oscillator system can also be inherited by an associated attractor [56] (chaotic or otherwise). A harmonic forcing of the coupled IF oscillator system $(1 \rightarrow I(1+A \cos \omega t))$ may be used to demonstrate this, since for a single oscillator, the rotation number of the firing map is irrational for a Cantor set of positive Lesbegue measure for parameter values in the region $1-1 / I>A \geq 0$ [57].

The counterpart of this paper is a study of non-identical pulse-coupled oscillators lacking periodic boundary conditions. One may no longer be able to exploit the group structure of the system, but many of the ideas in this paper can be built upon. For instance, numerical continuation from phase-locked solutions is still possible and so is analysis via averaging. Indeed studies of chains of coupled oscillators have revealed the existence of frequency plateaus in which two or more pools of oscillators are phase-locked but oscillate at different frequencies [8] and oscillator death where the coupling can actually destroy the oscillations completely $[58,59]$. In a subsequent paper 
[53] we prove the existence of phase-locked solutions for a chain of pulse-coupled IF oscillators with nearestneighbour delayed coupling using a singularly perturbed continuum boundary value formulation. In the large- $N$ limit phase-transitions are shown to occur upon variation of system parameters including the (different) natural frequencies of the oscillators.

\section{Acknowledgements}

The authors would like to acknowledge support from the EPSRC (UK) grant no. GR/K86220.

\section{References}

[1] A.T. Winfree, The Geometry of Biological Time, Springer, New York, 1980.

[2] Y. Kuramoto, Chemical Oscillations, Waves and Turbulence, Springer, Berlin, 1984.

[3] P. Hadley, M.R. Beasley, K. Wiesenfeld, Phase locking of Josephson-junction series arrays, Phys. Rev. B 38(13) (1998) $8712-8719$.

[4] S. Watanabe, S.H. Strogatz, Constants of motion for superconducting Josephson arrays, Physica D 74 (1994) 197-253.

[5] M. Silber, L. Fabiny, L. Wiesenfeld, Stability results for in-phase and splay-phase states of solid-state laser arrays, J. Opt. Sci. Am. B. 10(6) (1993) 1121-1129.

[6] K. Kurincsorgei, M. Orban, Oscillatory chemical-reactions in heterogeneous systems, J. Phys. Chem. 100(49) (1996) $19141-19147$.

[7] T.R. Chay, Bifurcations in heart rhythms, Int. J. Bifur. Chaos 5(6) (1995) 1439-1486.

[8] G.B. Ermentrout, N. Kopell, Frequency plateaus in a chain of weakly coupled oscillators., SIAM J. Math. Anal. 15 (1984) $215-237$.

[9] C.M. Gray, W. Singer, Stimulus-specific neuronal oscillations in orientation columns of cat visual-cortex., Proc. Natl. Acad. Sci. 86 (1989) $1698-1702$.

[10] J.C. Alexander, Patterns at primary Hopf bifurcations of a plexus of identical oscillators, SIAM J. Appl. Math. 46(2) (1986) $199-221$.

[11] N. Fenichel, Persistence and smoothness of invariant manifolds for flows, Indiana Univ. Math. J. 21 (1971) 193-266.

[12] J. Guckenheimer, P.J. Holmes, Nonlinear Oscillations, Dynamical Systems and Bifurcations of Vector Fields, Springer, New York, 1983.

[13] R.H. Rand, P.J. Holmes, Bifurcation of periodic motions in two weakly coupled van der pol oscillators, Int. J. Nonlinear Mech. 15 (1980) 387-399.

[14] N. Kopell, G.B. Ermentrout, Symmetry and phase-locking in chains of weakly coupled oscillators, Comm. Pure Appl. Math. 39 (1986) 623-660.

[15] N. Kopell, G.B. Ermentrout, Phase transitions and other phenomena in chains of coupled oscillators, SIAM J. Appl. Math. 50 (1990) $1014-1052$.

[16] F.C. Hoppensteadt, I. Izhekevich, Weakly connected neural nets, Applied mathematical sciences, vol. 126, Springer, New York, 1997.

[17] J.W. Swift, S.H. Strogatz, K. Wiesenfeld, Averaging of globally coupled oscillators, Physica D 55 (1992) 239-250.

[18] S. Watanabe, S.H. Stongatz, Integrability of a globally coupled oscillator array, Phys. Rev. Lett. 70(16) (1993) 2391-2394.

[19] D. Hansel, G. Mato, C. Meunier, Phase dynamics for weakly coupled Hodgkin-Huxley neurons, Europhys. Lett. 23(5) (1993) 367-372.

[20] D. Hansel, G. Mato, C. Meunier, Synchrony in excitatory neural networks, Neural Comput. 7 (1995) 307-337.

[21] P. Ashwin, J.W. Swift, The dynamics of $n$ weakly coupled identical oscillators, J. Nonlinear Sci. 2 (1992) 69-108.

[22] M. Golubitsky, I.N. Stewart, D.G. Schaeffer, Singularities and Groups in Bifurcation Theory, vol. 2, of Applied mathematical sciences, vol. 69, Springer, New York, 1988.

[23] G.P. King, P. Ashwin, J.W. Swift, Three identical oscillators with symmetric coupling, Nonlinearity 3 (1990) 585-601.

[24] J.J. Collins, I.N. Stewart, Coupled nonlinear oscillators and the symmetries of animal gaits, J. Nonlinear Sci. 3 (1993) 349-392.

[25] D.G. Aronson, M. Golubitsky, M. Krupa, Coupled arrays of Josephson junctions and bifurcations of maps with $\mathbf{S}_{n}$ symmetry, Nonlinearity 4(3) (1991) 861-902.

[26] H.C. Tuckwell Introduction to Theoretical Neurobiology, vol. I, Cambridge University Press, Cambridge, 1988.

[27] L.F. Abbott, T.B. Kepler, Model neurons: From Hodgkin-Huxley to Hopfield, in L. Garrido (Ed.), Statistical Mechanics of Neural Networks, Springer, Berlin, 1990, pp. 5-18.

[28] S. Bottani, Pulse-coupled relaxation oscillators: From biological synchronization to self-organized criticality, Phys. Rev. Lett. 74(21) (1995) 4189-4192.

[29] D. Chen, S. Wu, Z.R. Yang, Self-organized criticality in a cellular automaton model of pulse-coupled integrate-and-fire neurons, J. Phys. A 28 (1995) 5177-5182.

[30] A. Corral, C.J. Perez, A Diaz-Guilera, A. Arenas, Self-organized criticality and synchronization in a lattice model of integrate-and-fire oscillators, Phys. Rev. Lett. 74(1) (1995) 118-121.

[31] M. Usher, M. Stemmler, Dynamic pattern formation leads to $1 / f$ noise in neural population, Phys. Rev. Lett. 74(2) (1995) $326-329$.

[32] R.E. Mirollo, S.H. Strogatz, Synchronisation of pulse-coupled biological oscillators, SIAM J. Appl. Maths 50(6) (1990) $1645-1662$. 
[33] W. Gerstner, J.L. van Hemmen, Coherence and incoherence in a globally coupled ensemble of pulse-emitting units, Phys. Rev. Lett. 71(3) (1993) 312-315.

[34] M. Tsodyks, I. Mitkov, H. Sompolinsky, Pattern of synchrony in inhomogeneous networks of oscillators with pulse interactions, Phys. Rev. Lett. 71(8) (1993) 1280-1283.

[35] L.F. Abbott, C. van Vreeswijk, Asynchronous state in neural networks of pulse-coupled oscillators, Phys. Rev. E 48(2) (1993) 1483-1490.

[36] C. van Vreeswijk, Partial synchronization in populations of pulse-coupled oscillators, Phys. Rev. E 54(5) (1996) 5522-5537.

[37] W. Gerstner, J.L. van Hemmen, J. Cowan, What matters in neuronal locking?, Neural Comput. 8 (1996) 1653-1676.

[38] P.C. Bressloff, S. Coombes, B. De Souza, Dynamics of a ring of pulse-coupled oscillators: a group theoretic approach, Phys. Rev. Lett. 79 (1997) 2791-2794.

[39] C. van Vreeswijk, L.F. Abbott, G.B. Ermentrout, When inhibition not excitation synchronizes neural firing, J. Comput. Neurosci. 1 (1994) 313-321.

[40] A.M. Turing, The chemical basis of morphogenesis, Phil. Trans. Roy. Soc. London B 237 (1952) 37-72.

[41] G.B. Ermentrout, The behaviour of rings of coupled oscillators, J. Math. Biol. 23 (1985) 55-74.

[42] P.C. Bressloff, S. Coombes, Desynchronization, mode-locking and bursting in strongly coupled integrate-and-fire oscillators, Phys. Rev. Lett. 81 (1998) 2168-2171.

[43] P.C. Bressloff, S. Coombes, Spike train dynamics underlying pattern formation in integrate-and-fire oscillator networks, Phys. Rev. Lett. 81 (1998) 2384-2387.

[44] Ronald L. Calabrese, Half-center oscillators underlying rhythmic movements. in: M. A. Arbib, (Ed.), The Handbook of Brain Theory and Neural Networks, MIT Press, Cambridge, MA, pp. 444-447, 1995.

[45] S.M. Crook, G.B. Ermentrout, M.C. Vanier, J.M. Bower, The role of axonal delay in the synchronization of networks of coupled cortical oscillators, J. Comp. Neurosci. 4(2) (1997) 161-172.

[46] P.C. Bressloff, S. Coombes, Synchrony in an array of integrate-and-fire neurons with dendritic structure, Phys. Rev. Lett. 78 (1997) 4665-4668.

[47] J.J.B. Jack, D. Noble, R.W. Tsien, Electric Current Flow in Excitable Cells, Clarendon Press, Oxford, 1975.

[48] W. Rall, Theoretical significance of dendritic trees for neuronal input-output relations, in: R. Reiss (Ed.), Neural Theory and Modelling, Stanford University Press, Stanford, 1964.

[49] G.B. Ermentrout, XPP, Available from http://www2.pitt.edu/ phase/.

[50] U. Ernst, K. Pawelzik, T. Giesel, Synchronization induced by temporal delays in pulse-coupled oscillators, Phys. Rev. Lett. 74(9) (1995) $1570-1573$.

[51] S. Coombes, G.J. Lord, Desynchronisation of pulse-coupled integrate-and-fire neurons, Phys. Rev. E 55(3) (1996) 2104R-2107R

[52] S. Coombes, G.J. Lord, Intrinsic modulation of pulse-coupled integrate-and-fire neurons, Phys. Rev. E 56 (1997) 5809-5818.

[53] P.C. Bressloff, S. Coombes, Tavelling waves in chains of integrate-and-fire oscillators., Phys. Rev. Lett. 80 (1998) 4185-4188.

[54] D. Golomb, D. Hansel, B. Shraiman, H. Sompolinsky, Clustering in globally coupled phase oscillators, Phys. Rev. A 45(6) (1992) 35163530.

[55] G. Goldsztein, S.H. Strogatz, Stability of synchronization in networks of digital phase-locked loops, Int. J. Bifur. Chaos 5(4) (1995) 983-990.

[56] P. Ashwin, Symmetric chaos in systems of three and four forced oscillators, Nonlinearity 3 (1990) 603-617.

[57] J.P. Keener, F.C. Hoppensteadt, J. Rinzel, Integrate-and-fire models of nerve membrane response to oscillatory input, SIAM J. Appl. math. 41(3) (1981) 503-517.

[58] G.B. Ermentrout, N. Kopell, Oscillator death in systems of coupled neural oscillators, SIAM J. Math. Anal. 50 (1990) 125-146

[59] G.B. Ermentrout, N. Kopell, Multiple pulse interactions and averaging in systems of coupled neural oscillators, J. Math. Biol. 29 (1991) 195-217. 